\title{
Ist Österreich voll in die DM-Zone integriert? Eine ökonometrische Analyse*
}

\author{
Johannes Jaenicke \\ Fachbereich Wirtschaftswissenschaften, Universität Osnabrück \\ Reinhard Neck \\ Institut für Wirtschaftswissenschaften, Universität Klagenfurt
}

\begin{abstract}
Zusammenfassung: Seit Anfang der achtziger Jahre betreibt Österreich eine enge Anbindung seiner Währung an die DM, um bei der Inflationsbekämpfung von der Reputation der Deutschen Bundesbank zu profitieren. Als Ergebnis dieser Hartwährungspolitik wird in der Literatur angenommen, daß die österreichische Wirtschaft voll in die erweiterte DM-Zone integriert sei. Die vorliegende Arbeit konfrontiert diese Hypothese mit Daten. Insbesondere überprüft sie die deutsche Dominanzhypothese anhand von langfristigen Zinssätzen mit Kointegrations- und Granger-Kausalitätstests im vektorautoregressiven Modell. Hiernach werden die österreichischen Zinssätze nicht nur von den deutschen, sondern auch von den US-Zinssätzen beeinflußt. Außerdem kann entgegen der Erwartung keine Kointegrationsbeziehung gefunden werden. Die österreichische Währung ist damit (noch) nicht vollständig in die DM-Zone integriert.
\end{abstract}

\begin{abstract}
Since the early eighties, Austria has pegged its exchange rate to the deutschmark in order to gain from the German Bundesbank's reputation in fighting domestic inflation. Most authors assume that the Austrian economy is fully integrated into the enlarged DM-Zone as a result of this hard-currency policy. The present paper confronts this hypothesis with data and tests the German dominance hypothesis for long-term interest rates using cointegration and Granger causality analysis in a vector autoregressive model. Results show that Austrian interest rates are influenced not only by German interest rates, but also by those in the U.S. Moreover, no cointegration can be shown to exist between German and Austrian interest rates. Therefore, the Austrian currency is not yet fully integrated into the DM-Zone.
\end{abstract}

Schlüsselwörter: Währungspolitik, internationaler Zinszusammenhang, deutsche Dominanzhypothese, Granger-Kausalität, Kointegration.

\section{$1 \quad$ Einleitung}

Das im Vertrag von Maastricht festgelegte und seither durch zahlreiche politische Absichtserklärungen bestätigte Ziel der Bildung einer Europäischen Wirtschafts- und Währungsunion (EWU) stellt für die Mitgliedsländer der Europäischen Union eine 
beträchtliche Herausforderung dar. Infolge der durch die rezessiven Entwicklungen der Weltwirtschaft verursachten budgetären Probleme der meisten Mitgliedstaaten der EU steht dabei in der wirtschaftspolitischen Diskussion derzeit die Erfüllung der fiskalischen Konvergenzkriterien im Mittelpunkt. Zumindest für die Länder, die bereits seit einigen Jahren einen festen Wechselkurs ihrer Währungen zur DM halten konnten, gelten dagegen die Zinssatz- und Inflationskriterien als unproblematisch, da angenommen wird, daß diese Volkswirtschaften bereits in eine DM-Zone integriert sind und ihre Geldpolitik weitgehend jener der Deutschen Bundesbank untergeordnet haben, so daß sie wohl ohne Schwierigkeiten auch der Geldpolitik der nach deutschem Vorbild konstruierten Europäischen Zentralbank folgen werden. Dies gilt nicht nur für einige Kernländer der EU, wie die Niederlande, Luxemburg, Belgien und Frankreich, die im Rahmen des Europäischen Währungssystems weitgehend feste Währungsrelationen zur DM verwirklicht haben, sondern auch (möglicherweise noch stärker) für Österreich, das zwar erst zum 1. Januar 1995 der EU und dem EWS beigetreten ist, aber bereits seit langem als "Schattenmitglied" des EWS autonom im Rahmen seiner "Hartwährungspolitik" einen faktisch unveränderten Schilling-DM-Wechselkurs realisiert hat. Es wird daher oft behauptet, daß Österreich voll in die DM-Zone integriert sei.

In der vorliegenden Arbeit wird diese in der einschlägigen Literatur fast unbestrittene Annahme näher untersucht. Im zweiten Abschnitt stellen wir die Entwicklung der österreichischen Hartwährungspolitik kurz dar und diskutieren einige mögliche Auswirkungen dieser Politik. Anschließend wird mit Hilfe von neueren ökonometrischen Verfahren überprüft, ob sich die Integration der österreichischen in die deutsche Wirtschaft empirisch nachweisen läßt. Ausgangspunkt ist dabei die deutsche Dominanzhypothese bezüglich der Geldpolitik, die für die Finanzmärkte anhand der internationalen Zinszusammenhänge getestet werden kann. Der dritte Abschnitt der Arbeit formuliert die zu testenden Hypothesen, während im vierten Abschnitt erste empirische Evidenz in Form von Kointegrations- und Granger-Kausalitätstests geliefert wird. Wir verwenden dabei langfristige Zinssätze, deren Konvergenz als Voraussetzung für die EWU im Vertrag von Maastricht gefordert wird.

Dabei erhalten wir überraschende Ergebnisse: Es kann keine Kointegration zwischen deutschen und österreichischen langfristigen Zinssätzen nachgewiesen werden; eine langfristige Gleichgewichtsbeziehung scheint also nicht vorzuliegen. Die Kausalitätstests zeigen zwar erwartungsgemäß den Einfluß der deutschen auf die österreichischen Zinssätze auf, ebenso wird jedoch ein unabhängiger Einfluß der US-Zinssätze auf die Entwicklung der österreichischen Zinssätze nachgewiesen. Diese empirischen Befunde deuten darauf hin, daß die österreichische Währung trotz der Hartwährungspolitik der Oesterreichischen Nationalbank noch nicht vollständig in die DM-Zone integriert ist.

\section{2 Österreichs Hartwährungspolitik}

Die Wechselkurspolitik Österreichs seit den frühen siebziger Jahren kann mit dem Begriff der "Hartwährungspolitik" charakterisiert werden. Die Oesterreichische Nationalbank war und ist bestrebt, den Wechselkurs des Schillings an andere "harte" Währungen anzubinden. Als "harte" Währungen werden dabei die Währungen jener 
Länder gesehen, die im internationalen Vergleich geringe Inflationsraten aufweisen, insbesondere die DM. Eine Freigabe des Schilling-Wechselkurses wurde als wirtschaftspolitische Option nie ernsthaft in Erwägung gezogen.

Die Hartwährungspolitik bildete sich zunächst als ein Bestandteil der wirtschaftspolitischen Konzeption des "Austrokeynesianismus" heraus. Im Laufe der achtziger Jahre wurde die Konzeption des Austrokeynesianismus zunehmend in Zweifel gezogen, teilweise infolge von wirtschaftspolitischen Neuorientierungen auf der internationalen Ebene, teilweise wegen der als unzureichend angesehenen Wirksamkeit einzelner seiner Instrumente. Der Niedergang des Austrokeynesianismus betrifft jedoch nicht die Hartwährungspolitik; diese wurde vielmehr bis jetzt konsequent weiterverfolgt.

Entsprechend der mehr "spontanen" als geplanten Herausbildung der Hartwährungspolitik wurden die mit ihr angestrebten Ziele nicht in kanonischer Form offiziell deklariert. Es ist jedoch unbestritten, daß durch die Stabilisierung des Außenwerts des Schillings ein Beitrag zur Verminderung von Unsicherheit bezüglich der Wechselkurs- und Preiserwartungen geleistet werden soll. Ein weiteres Hauptziel dieser Politik ist die Förderung der Preisstabilität durch den "Import" niedriger Inflationsraten, insbesondere aus der Bundesrepublik Deutschland. Daneben wird häufig das langfristige Ziel der Verbesserung der Wettbewerbsfähigkeit der österreichischen Volkswirtschaft mit der Hartwährungspolitik in Verbindung gebracht.

Die Entwicklung der österreichischen Wechselkurspolitik kann folgendermaßen kurz charakterisiert werden. ${ }^{1}$ Die Hartwährungspolitik wurde zu Beginn der siebziger Jahre zuerst eingeführt, um die Inflation und spekulative Kapitalbewegungen zu bekämpfen. Nach dem Zusammenbruch des Bretton-Woods-Systems verfolgte die Oesterreichische Nationalbank zunächst eine Politik des "managed floating". Dabei wurden zunächst verschiedene Orientierungsgrößen für den Wechselkurs des österreichischen Schillings verwendet, insbesondere Wechselkursindizes.

Im Gefolge des ersten Ölpreisschocks (1974 bis 1976) reagierten die Fiskal- und die Einkommenspolitik in Österreich expansiv; der Schilling wertete zugleich gegenüber den Haupthandelspartnern auf. Diese Politik führte zu schwerwiegenden Leistungsbilanzproblemen. Restriktive Maßnahmen mußten ergriffen werden, um das Leistungsbilanzdefizit abzubauen. Gleichzeitig versuchte die österreichische Geldpolitik, die Zinssätze auf niedrigem Niveau zu halten, um Investitionen zu erleichtern. Im Zuge der beginnenden Hochzinsphase geriet diese Politik der Nominalzinskonstanz in Widerspruch zur Hartwährungspolitik. Dieser Zielkonflikt wurde zugunsten der Wechselkursstabilität gelöst: Bei zunehmender Kapitalsubstitution und internationaler Integration der österreichischen Finanzmärkte war die Oesterreichische Nationalbank 1979 gezwungen, die Zinssätze nach oben anzupassen.

In den folgenden Jahren wertete der österreichische Schilling zwar gegenüber dem US-Dollar zunächst ab, beteiligte sich aber an allen Wechselkursanpassungen der DM innerhalb des Europäischen Währungssystems, wobei er gegenüber dem ECU und von 1979 bis 1981 sogar gegenüber der DM aufwertete. Die Signalwirkung dieser Aufwertung wurde in der Literatur stark hervorgehoben; vgl. WINCKLER (1991). Seit Ende 1981 ist der DM-Wechselkurs des Schillings weitgehend konstant. 
Nach dem zweiten Ölpreisschock war die österreichische Wirtschaftspolitik von 1981 bis 1983 zunächst wieder eher expansiv ausgerichtet. Ab 1984 lagen die österreichischen Zinssätze über jenen der Bundesrepublik Deutschland, da Österreich eine etwas höhere Inflation aufwies. Aufgrund der im März 1985 einsetzenden Abwertung des US-Dollars wertete der Schilling real wieder auf, da nicht mehr der reale effektive Wechselkurs des Schillings, sondern der Schilling-DM-Wechselkurs konstant gehalten wurde. In diesen Jahren verstärkte sich die Bindung des Schillings an die DM sogar noch weiter; die Schwankungen des Schilling-DM-Wechselkurses verschwanden fast völlig.

Diese Bindung des österreichischen Schillings an die DM wurde auch durch die deutsche Vereinigung und durch die Krisen des Europäischen Währungssystems 1992 und 1993 nicht beeinträchtigt. Trotz Liberalisierung des Kapitalverkehrs und Öffnung der Finanzmärkte in Österreich hatten Spekulationen gegen den Schilling auch in dieser Periode keine nennenswerte Bedeutung. Dies wird im allgemeinen dahingehend interpretiert, daß die österreichische Wirtschaft bereits voll in die DM-Zone integriert ist, zu der außer Deutschland meist auch die Benelux-Länder und Frankreich gezählt werden.

Die wichtigste und fast allgemein anerkannte Begründung für die österreichische Hartwährungspolitik liegt in ihrer inflationsvermindernden Wirkung. Die einschlägige Literatur stimmt dahingehend überein, daß die österreichische Hartwährungspolitik und die dadurch bewirkte weitgehende Integration Österreichs in die DM-Zone das Ziel der Inflationsverminderung im wesentlichen erreicht habe. So lag die österreichische Inflationsrate in den siebziger und achtziger Jahren unter dem Durchschnitt der OECD-Länder und war auch niedriger als jene der wichtigsten Handelspartner Österreichs. Eine Ausnahme stellt die Inflationsrate der Bundesrepublik Deutschland dar, die in den meisten Jahren der Periode der Hartwährungspolitik geringer war als jene Österreichs. Das Inflationsdifferential zwischen beiden Ländern dürfte unter anderem auf die expansivere Fiskalpolitik Österreichs zurückzuführen sein. Diese Politik hatte jedoch auch (zumindest kurzfristig) reale Effekte $^{2}$ und hat dazu beigetragen, daß die Wachstumsraten des österreichischen Brutto-Inlandsprodukts bis in die Mitte der achtziger Jahre höher waren als jene der Bundesrepublik Deutschland. Als Nebenwirkung der Kombination aus Hartwährungspolitik und expansiver Fiskalpolitik lagen die österreichischen Nominalzinssätze meist über jenen der Bundesrepublik Deutschland.

Als theoretische Begründung für die preisstabilisierende Wirkung der Hartwährungspolitik wurde in Österreich zunächst meist die Kostendruckerklärung von Inflationen herangezogen. Dabei werden die Importpreise und die Lohn- und Preispolitik der Gewerkschaften und der Arbeitgeber (und ihrer Interessenorganisation) im Rahmen des Systems der Wirtschafts- und Sozialpartnerschaft als die wichtigsten Bestimmungsgrößen des österreichischen Preisniveaus angesehen. Allerdings sollte nicht übersehen werden, daß auch eine am Zwischenziel der Geldmengensteuerung orientierte restriktive Politik der Oesterreichischen Nationalbank ähnliche (vielleicht sogar stärkere) inflationsdämpfende Wirkungen hätte haben können wie die am Zwischenziel des Wechselkurses ausgerichtete Hartwährungspolitik. Der spezifisch österreichische 
"policy-mix" und das institutionelle Arrangement, auf dem dieser beruht, wurden unter anderem durch strategische Aspekte der beteiligten Entscheidungsträger erklärt.

Auf diese strategischen Elemente in den Interaktionen zwischen Fiskal-, Geld- und Einkommenspolitik wurde vor allem in den Arbeiten von WINCKLER ${ }^{3}$ hingewiesen. Dabei wird die Hartwährungspolitik als ein Mittel dafür angesehen, die Wirtschafts- und Sozialpartner zu mäßigeren Lohn- und Preisforderungen zu veranlassen. Allerdings bleibt dabei die Frage offen, warum nicht durch eine kooperative Wirtschaftspolitik, auf die sich Regierung, Notenbank und Wirtschafts- und Sozialpartner festlegen, mit Hilfe von diskretionären Anpassungen des Wechselkurses bei vom Ausland kommenden Schocks bessere Ergebnisse erzielt werden als mit der "fixierten Regel" der österreichischen Hartwährungspolitik.

Eine Antwort darauf kann in dem Argument der Zeitinkonsistenz einer solchen "optimalen" Wirtschaftspolitik gefunden werden. Demnach können "fixierte Regeln" einen höheren Grad an Glaubwürdigkeit für die Wirtschaftspolitik bewirken als diskretionäre Änderungen, insbesondere in Hinblick auf das Ziel der Geldwertstabilität. Obwohl die Annahmen über die makroökonomischen Zusammenhänge und die Modellierung der strategischen Interaktionen zwischen den Regierungen, den Notenbanken und den privaten Akteuren der Volkswirtschaften, die diesem Argument zugrunde liegen, keineswegs unbestritten sind, geht doch ein Großteil der theoretischen Literatur zur europäischen Währungspolitik von einem derartigen Ansatz aus. Dabei wird gezeigt, daß es unter bestimmten Voraussetzungen für andere europäische Länder vorteilhaft sein kann, ihre geldpolitische Autonomie aufzugeben und sich der Geldpolitik der Deutschen Bundesbank unterzuordnen. Wenn dies in glaubwürdiger Weise möglich ist, kann damit die Reputation der deutschen Geldpolitik als "Anker" der Inflationsbekämpfung auf die jeweilige Notenbank übertragen und dadurch eine erfolgreiche Stabilitätspolitik implementiert werden. Vgl. dazu u.a. GIAVAZZI und GiOVANNINI (1989).

Für eine kleine offene Volkswirtschaft wie die österreichische kann die Fixierung des Wechselkurses zur DM eine besonders glaubwürdige Regel darstellen, die relativ einfach durchschaubar und durchsetzbar ist. HOCHREITER und WINCKLER (1995) interpretieren die Hartwährungspolitik der Oesterreichischen Nationalbank seit den späten siebziger Jahren in diesem Sinn und demonstrieren modellmäßig und datenanalytisch, daß diese Politik dazu geführt haben kann, daß Österreich in den achtziger Jahren Teil eines optimalen Währungsraums mit Deutschland geworden ist, wobei trotz teilweise asymmetrischer Schocks auf beide Volkswirtschaften und begrenzter Faktormobilität die durch die Hartwährungspolitik induzierte erhöhte Reallohnflexibilität für die Integration der österreichischen Volkswirtschaft in die optimale Währungszone mit Deutschland verantwortlich gemacht wird. 


\section{Zur Operationalisierung der deutschen Dominanz- hypothese für Österreich}

Die Interpretation der österreichischen Hartwährungspolitik als Ursache für eine Integration Österreichs in die DM-Zone wirft eine Fülle von theoretischen und insbesondere empirischen Problemen auf, die hier nicht im Detail behandelt werden können. Wir beschränken uns vielmehr auf einen, für diese These allerdings wesentlichen Teilaspekt, und zwar auf die Frage, ob die österreichische Geldpolitik tatsächlich von der deutschen Geldpolitik (und nur von dieser) bestimmt wurde und ob sich diese Dominanz der deutschen Geldpolitik im Verhalten der Finanzmärkte und insbesondere in der Entwicklung des österreichischen Zinssatzes seit Beginn der achtziger Jahre nachweisen läßt.

Theoretische Grundlage für die Hypothesen über die Zinszusammenhänge zwischen Österreich und Deutschland bildet die Theorie der ungedeckten Zinsparität. Diese setzt voraus, daß inländische und ausländische Wertpapiere vollkommene Substitute sind, was unter anderem fehlende Transaktionskosten, freien Kapitalverkehr und gleiche Risikoeigenschaften in- und ausländischer Kapitalanlagen erfordert. In diesem Fall wird der inländische Zinssatz durch den ausländischen Zinssatz und die erwartete Wechselkursänderung bestimmt. Bei Gültigkeit der deutschen Dominanzhypothese müßte demnach der österreichische Zinssatz durch den deutschen Zinssatz determiniert sein, da bei glaubwürdiger Fixierung des Schilling-DM-Wechselkurses von den privaten Wirtschaftssubjekten keine Wechselkursänderungen erwartet werden; gleiches gilt übrigens, wenn der Wechselkurs durch einen Random Walk beschrieben werden kann. Kurz- oder langfristige Abweichungen der österreichischen von den deutschen Zinssätzen können insbesondere auftreten, wenn durch unterschiedliche Inflationserwartungen für beide Länder Risikoprämien für erwartete Wechselkursänderungen vorliegen. Bei vollständiger Integration der österreichischen und deutschen Kapitalmärkte müßten diese Risikoprämien allerdings verschwinden; zumindest muß Stationarität der Risikoprämien und damit eine langfristige Gleichgewichtsbeziehung zwischen österreichischen und deutschen Zinssätzen erwartet werden. ${ }^{4}$

Die deutsche Dominanzhypothese wurde (mit unterschiedlichen Ergebnissen) in der Literatur für verschiedene Mitgliedsländer des EWS anhand der Zinssätze empirisch überprüft. Für unsere Untersuchung der Gültigkeit dieser Hypothese für das EWS"Schattenmitglied" Österreich verwenden wir im Anschluß an KIRCHGÄSSNER und Wolters (1993, 1995) die Methodologie der Tests auf Granger-Kausalität und auf Kointegration. Granger-Kausalität gibt Aufschluß über kurzfristige Beziehungen zwischen Zinssätzen verschiedener Länder ("kurzfristige Dominanzhypothese"). Wenn die Zinssätze nicht stationär sind, wie es häufig der Fall ist, erfordert das Bestehen langfristiger Gleichgewichtsbeziehungen darüber hinaus Kointegration zwischen den Zinssätzen ("langfristige Dominanzhypothese"). Liegt keine derartige Beziehung zwischen dem österreichischen und dem deutschen Zinssatz vor, so kann selbst bei durch Granger-Kausalität nachzuweisender kurzfristiger Dominanz des österreichischen durch den deutschen Zinssatz die Oesterreichische Nationalbank die langfristige Entwicklung des österreichischen Zinssatzes noch beeinflussen. Eine vollständige Integration der 
österreichischen Volkswirtschaft in die DM-Zone setzt also das Bestehen sowohl von Granger-Kausalitätsbeziehungen wie von Kointegrationsbeziehungen zwischen deutschem und österreichischem Zinssatz und nur zwischen diesen voraus.

Ausgangspunkt für die Tests der deutschen Dominanzhypothese bezüglich des österreichischen Zinssatzes sind die Teilhypothesen, die VON HAGEN und FRATIANNI (1990) für die EWS-Mitgliedsländer formuliert haben. In Hinblick auf Granger-Kausalität lauten diese:

H1 (Abhängigkeit von Deutschland): Der deutsche Zinssatz ist granger-kausal für den österreichischen Zinssatz.

H2 (Unabhängigkeit Deutschlands von Österreich): Der österreichische Zinssatz ist nicht granger-kausal für den deutschen Zinssatz.

H3 (Isolierung Österreichs vom Rest der Welt): Zinssätze anderer Länder als Deutschlands sind nicht granger-kausal für den österreichischen Zinssatz.

Die deutsche Dominanzhypothese für Österreich (die vollständige Integration Österreichs in die DM-Zone) verlangt die gemeinsame Gültigkeit aller drei Hypothesen, also eine Kausalitätsbeziehung (H1) und zwei Nicht-Kausalitätsbeziehungen (H2 und H3). Als Repräsentanten für den "Rest der Welt" betrachten wir im folgenden nur den Zinssatz der USA.

Da Granger-Kausalität nicht Kointegration impliziert, muß das Bestehen langfristiger Gleichgewichtsbeziehungen für die Zinssätze zusätzlich durch Kointegrationstests überprüft werden, wenn die Zinssätze nichtstationär sind. Bei Bestehen von Kointegrationsbeziehungen existieren Fehler-Korrektur-Darstellungen zwischen den entsprechenden Zinssätzen. Die Hypothese der langfristigen Dominanz Deutschlands kann dann nach KIRCHGÄSSNER und WOLTERS (1993, S. 774) folgendermaßen formuliert werden:

H1': Der deutsche Zinssatz ist im Fehler-Korrektur-Term der Gleichung für den österreichischen Zinssatz enthalten.

H2': Der österreichische Zinssatz ist nicht im Fehler-Korrektur-Term der Gleichung für den deutschen Zinssatz enthalten.

H3': Zinssätze anderer Länder als Deutschlands, die nicht dem EWS angehören, sind nicht im Fehler-Korrektur-Term der Gleichung für den österreichischen Zinssatz enthalten.

Bei einem gemeinsamen Test dieser drei Hypothesen in einem vektorautoregressiven Modell in Fehler-Korrektur-Darstellung für den österreichischen, den deutschen und einen weiteren Zinssatz verlangt die Gültigkeit der langfristigen deutschen Dominanzhypothese, daß zwischen Deutschland und Österreich in einem bivariaten Modell genau ein Kointegrationsvektor existiert. Wird ein weiteres Land berücksichtigt, das nicht dem EWS angehört, so muß mindestens ein Kointegrationsvektor vorliegen. Auch hier wird als weiteres Land wieder nur die USA untersucht; Erweiterungen auf andere für das Portefeuille-Verhalten der österreichischen Anleger möglicherweise relevante Währungen sind für weitere Untersuchungen geplant. 


\section{Empirische Ergebnisse}

\subsection{Vorgehen und Datenauswahl}

Um die Hypothese der Integration Österreichs in die DM-Zone zu überprüfen, werden in dieser Arbeit die Umlaufrendite öffentlicher Anleihen in Österreich (ALR) und in Deutschland $(\mathrm{GLR})^{5}$ sowie die Umlaufrendite von US-Governmentbonds mit einer Restlaufzeit von zehn Jahren (ULR) verwendet. ${ }^{6}$ Da der Kurs des Schillings seit 1981 weitgehend an die DM angebunden ist, beginnt der Untersuchungszeitraum im Januar 1981 und endet im November 1994, also kurz vor dem Beitritt Österreichs zum Europäischen Währungssystem. Die Zinssätze sind in Abbildung 1 wiedergegeben.

Abbildung 1: Langfristige Zinssätze in Österreich, Deutschland und den USA Zeitraum: Januar 1981 bis November 1994

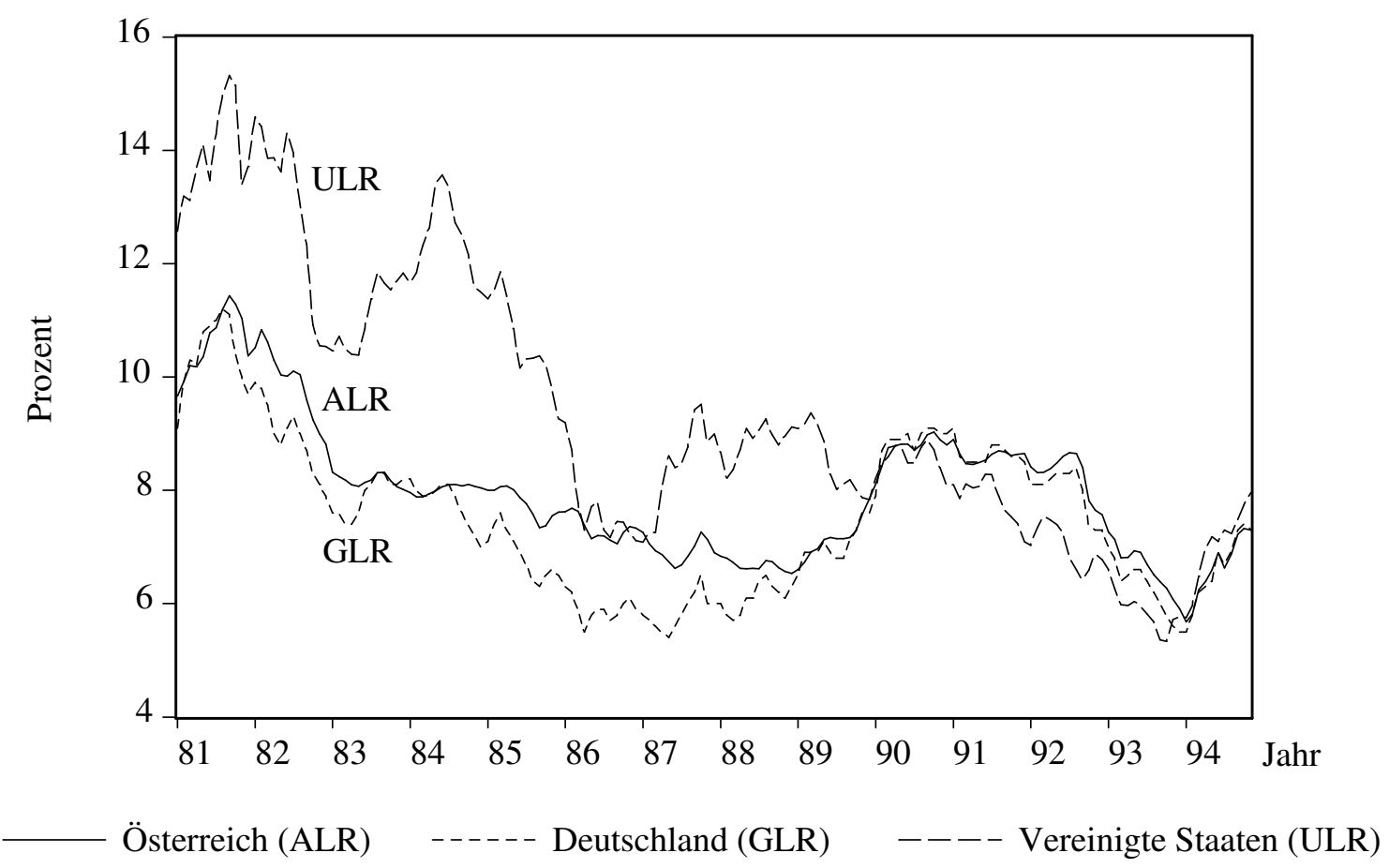

In früheren Untersuchungen zum Einfluß der DM auf Währungen im Europäischen Währungssystem wurden häufig kurzfristige Geldmarktsätze verwendet, da sie als stärker von den jeweiligen Zentralbanken beeinflußbar angesehen werden (vgl. z. B. vON HAGEN und FratianNi (1990) und KIRCHGÄSSNER und WOLTERS (1995)). Allerdings fordert der Vertrag von Maastricht die Konvergenz der langfristigen Zinssätze als ein Konvergenzkriterium für die Mitgliedsländer der zukünftigen Europäischen Wirtschafts- und Währungsunion. In langfristigen Zinssätzen kommen die erwartete Inflationsrate und die jeweilige Risikoprämie und damit das Verhalten der Akteure auf den Kapitalmärkten stärker zum Ausdruck, so daß ein enger und dauerhafter 
Zusammenhang zwischen den deutschen und österreichischen langfristigen Zinssätzen für einen engen Zusammenhang der beiden Währungen spricht. Werden Impulse von den internationalen Märkten (z. B. vom US-Kapitalmarkt) zunächst in den deutschen und dann erst in den österreichischen Zinsen reflektiert, so kommt hierin ein asymmetrisches Verhältnis der beiden Währungen zum Ausdruck: die DM spielt eine dominierende Rolle, und der österreichische Schilling ist nur an sie gekoppelt. Die Frage nach dem langfristigen Zusammenhang wird hier mit Kointegrationstests untersucht, die nach dem Schockverarbeitungsmechanismus mit GrangerKausalitätstests. Für beide Testverfahren ist zunächst die Stationarität der Zinssätze zu überprüfen.

\subsection{Ergebnisse der Stationaritäts- und Kointegrationstests}

TIEDE (1991, S.100) weist in seiner Kritik an Granger-Kausalitätstests darauf hin, daß diese Tests schwach stationäre Zeitreihen voraussetzen. Ein stochastischer Prozeß wird (schwach) stationär genannt, wenn sein Erwartungswert und seine Autokovarianzen keine Funktionen der Zeit sind. Ein nichtstationärer Prozeß heißt integriert von der Ordnung eins, wenn die erste Differenz dieses Prozesses stationär ist. Sind dagegen die Zeitreihen kointegriert, d. h. es existiert zwischen den von der Ordnung eins integrierten Variablen eine nicht-triviale stationäre Linearkombination, dann sind direkt mit den integrierten Zeitreihen Granger-Kausalitätstests durchzuführen (vgl. SIMS, STOCK und WATSON (1990) und TODA und PHILliPS (1994)). In diesem Fall existiert nach dem Granger-Repräsentationstheorem (ENGLE und GRANGER (1987, S. 255 f.)) eine FehlerKorrektur-Darstellung der Variablen, und es liegt Granger-Kausalität in (mindestens) eine Richtung vor. Vektorautoregressive Modelle in ersten Differenzen wären in diesem Fall fehlspezifiziert.

Um empirisch die Frage der Stationarität zu überprüfen, wird nach dem erweiterten Dickey-Fuller-Test mit der Methode der kleinsten Quadrate (OLS) folgende Gleichung geschätzt:

$$
\Delta Y_{t}=\beta_{0}+(\rho-1) Y_{t-1}+\sum_{j=1}^{k} \gamma_{j} \Delta Y_{t-j}+U_{t}
$$

Dabei bezeichnet $Y_{t}$ die jeweils benutzte Variable ALR, GLR und ULR, $\Delta Y_{t}:=Y_{t}-Y_{t-1}$ die erste Differenz der Zeitreihe und $U_{t}$ die Störung. Die Nullhypothese $H_{0}:|\rho|=1$ besagt, daß $Y_{t}$ integriert von der Ordnung eins ist und damit nichtstationäres Verhalten aufweist. Kann die Nullhypothese zugunsten von $H_{1}:|\rho|<1$ verworfen werden, so ist $Y_{t}$ stationär. Die Teststatistik $t_{(\rho-1)}$ für den Koeffizienten $(\rho-1)$ ist Fuller-verteilt ${ }^{7}$. Da die Tests im Fall $k=1$ erheblich verzerrt sein können, wenn die Zeitreihe $Y_{t}$ eine moving averageKomponente erster Ordnung (MA(1)) mit negativem Koeffizienten enthält (vgl. SCHWERT (1989, S. 152) und HASSLER (1993, S. 70)), wurde anstelle von Gleichung (1) gemäß SAID und DICKEY (1985) auch eine moving average-Formulierung benutzt:

$$
\Delta Y_{t}=\beta_{0}+(\rho-1) Y_{t-1}+U_{t}+\alpha U_{t-1} .
$$

Der Einsatz von Modellselektionskriterien erhöht nach HALL (1994) die Macht des erweiterten Dickey-Fuller-Tests. Mit Hilfe des AIC-, SC- und HQ-Selektionskriteriums 
(vgl. z. B. JUDGE u. a. (1985, S. 244-247)) wurde daher eine Auswahl zwischen Gleichung (1) und (2) getroffen. ${ }^{8}$ Die maximal mögliche Anzahl der Lags in Gleichung (1) wurde mit $k=6$ festgelegt. Der Dickey-Fuller-Test unterstellt unabhängig identisch normalverteilte Residuen. Daher wurden die Residuen in Gleichung (1) bzw. (2) mit der Durbin-Watson-Statistik ${ }^{9}$ und der Ljung-Box-Q(12)-Statistik auf Autokorrelation erster und höherer Ordnung überprüft. Dabei ist nach MACKINNON (1994, S. 167) die Unkorreliertheit der Residuen wesentlich, im Gegensatz zur Homoskedastizitäts- und Normalverteilungsannahme, die mit dem Engle-Test auf ARCH(1)-Residuen und der JarqueBera-Statistik überprüft wurden.

Die Ergebnisse der Tests auf Stationarität finden sich in Tabelle 1. Die Nullhypothese der Einheitswurzel kann in keinem Fall verworfen werden. ${ }^{10}$ Aufgrund der gefundenen Evidenz gegen die Homoskedastizitäts- und Normalverteilungsannahme wurde zusätzlich der von PHILLIPS (1987) und PHILLIPS und PERRON (1988) vorgeschlagene $Z_{\rho^{-}}$ und $Z_{t}$-Test durchgeführt, da dort schwächere Anforderungen an die Residuen gestellt werden. ${ }^{11}$ Die Anzahl der Lags beim $Z_{\rho}$ - und $Z_{t}$-Test wurde wie bei SCHWERT (1987, S. 88) abhängig vom Beobachtungsumfang gemäß dem Kriterium $l=\operatorname{Int}\left\{4(T / 100)^{1 / 4}\right\}$ festgelegt und beträgt $l=4$. Die vorliegenden Ergebnisse des Phillips-Perron-Tests, ebenfalls in Tabelle 1, sind sehr eindeutig: Die Hypothese der einfachen Einheitswurzel kann für die drei Zinssätze auf keinem üblichen Signifikanzniveau verworfen werden. Damit muß von der Nichtstationarität der betrachteten Zinssätze ausgegangen werden.

Um zu prüfen, ob die drei Zeitreihen integriert von der Ordnung eins sind, wurden der erweiterte Dickey-Fuller- und der Phillips-Perron-Test mit der ersten Differenz der Zeitreihen durchgeführt. Die Testergebnisse sind in Tabelle 1 wiedergegeben. Beim Dickey-Fuller-Test wird die Nullhypothese der zweifachen Einheitswurzel mit einer Irrtumswahrscheinlichkeit von höchstens 1 Prozent abgelehnt. Allerdings werden für den US-Zinssatz hochsignifikante ARCH-Effekte und Abweichungen von der Normalverteilungsannahme nachgewiesen. Jedoch bestätigt der Phillips-Perron-Test die Ergebnisse des Dickey-Fuller-Tests: Die Nullhypothese wird stets auf dem 1-Prozent-Signifikanzniveau verworfen, so daß, insgesamt betrachtet, die drei langfristigen Zinssätze als integriert von der Ordnung eins angesehen werden können. Dies kann zu Scheinregressionsbeziehungen führen, wie PHILLIPS (1986) zeigt.

Um trotz der nachgewiesenen Nichtstationarität statistische Aussagen über die langfristige Integration der österreichischen Zinssätze in die erweiterte DM-Zone machen zu können, wird das von GRANGER (1986) und ENGLE und GRANGER (1987) entwickelte Konzept der Kointegration benutzt. Die von der Ordnung eins integrierten Zinssätze heißen kointegriert, wenn es eine Linearkombination $U=Y-\alpha-X \beta$ gibt, die stationär ist. Zum Test auf Kointegration werden in dieser Arbeit univariate und multivariate Verfahren benutzt.

Gemäß dem Vorschlag von ENGLE und GRANGER (1987, S. 261) wird die sogenannte einfache kointegrierende Gleichung mit der Methode der kleinsten Quadrate (OLS) geschätzt: ${ }^{12}$

$$
Y_{t}=\alpha+\beta_{1} X_{1, t}+\beta_{2} X_{2, t}+U_{t},
$$

und dann überprüft, ob die Störungen $U_{t}$ stationär sind oder nicht. Hierzu werden der 


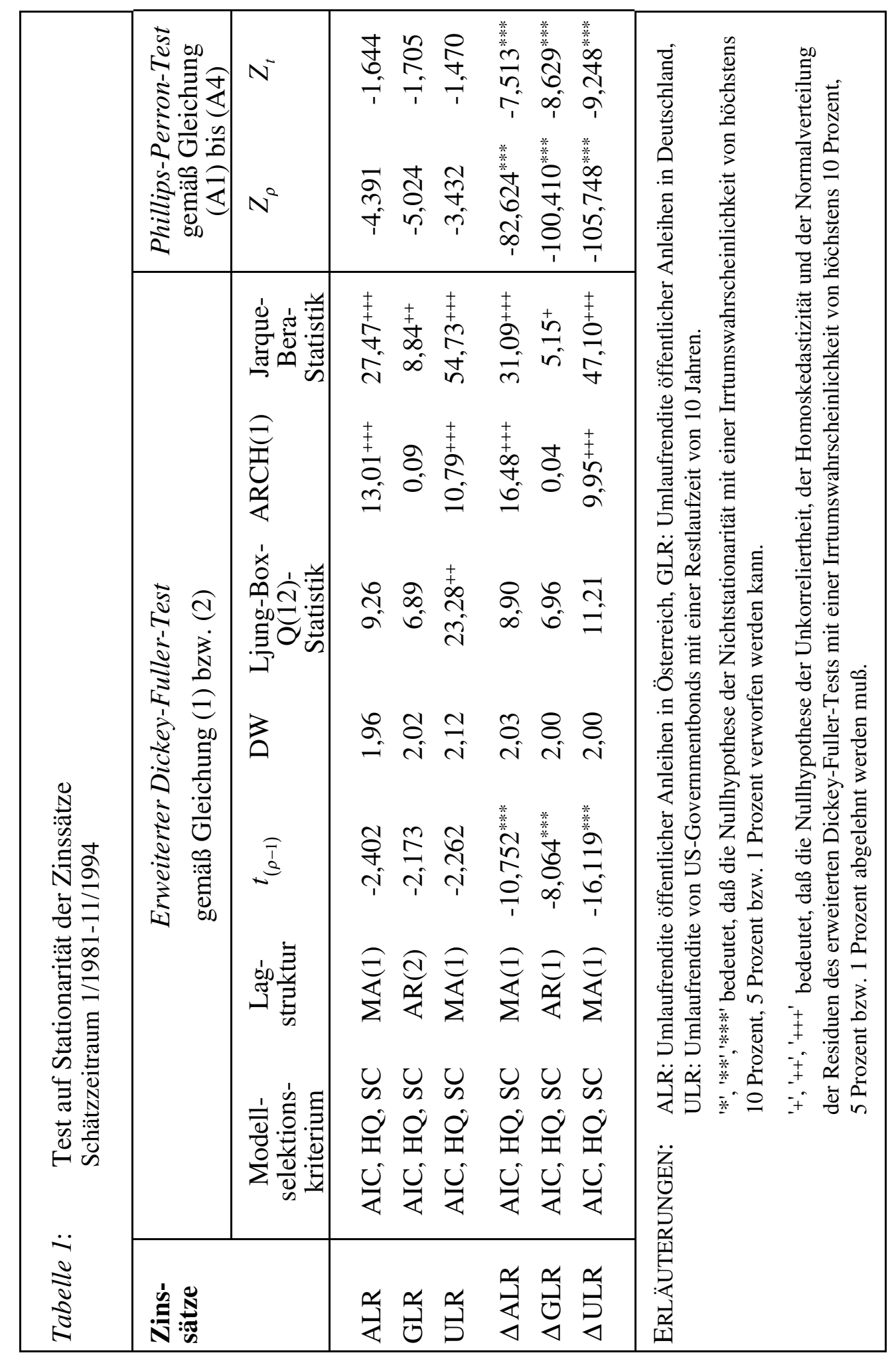


von Engle und Granger vorgeschlagene Durbin-Watson-Test und der erweiterte DickeyFuller-Test benutzt sowie die von PHILLIPS und Ouliaris (1990) vorgeschlagenen $Z_{\rho^{-}}$ und $Z_{t}$-Tests. Alle vier Kointegrationstests postulieren als Nullhypothese, daß $Y, X_{1}$ und $X_{2}$ nicht kointegriert sind.

Im ersten Test wird gefragt, ob der Wert der Durbin-Watson-Statistik (CRDW) der kointegrierenden Gleichung signifikant größer als null ist. ${ }^{13}$ Neben diesem einfachen Test findet der erweiterte Dickey-Fuller-Test (ADF) Anwendung. Hierzu wird mit den Störungen $U_{t}$ der einfachen kointegrierenden Gleichung der erweiterte Dickey-FullerEinheitswurzeltest gemäß Gleichung (1) und (2) unter der Restriktion $\beta_{0}=0$ durchgeführt. Die maximal mögliche Anzahl der Lags in Gleichung (1) wurde wieder mit $k=6$ vorgegeben. Die Nullhypothese der Nichtkointegration, $H_{0}:|\rho|=1$, wird anhand des Wertes der t-Statistik von $(\rho-1)$ überprüft. ${ }^{14}$ Aufgrund der häufig nachgewiesenen $\mathrm{Ab}$ weichungen von der Homoskedastizität und Normalverteilung wird zusätzlich der $Z_{\rho^{-}}$ und $Z_{t}$-Test von Phillips-Ouliaris angewendet. ${ }^{15}$ Die Ergebnisse dieser Tests sind in Tabelle 2a wiedergegeben. Die Nullhypothese der Nicht-Kointegration der Zinssätze kann für keine der möglichen Kombinationen der drei Zinssätze auf einem üblichen Signifikanzniveau verworfen werden. Dabei muß allerdings beachtet werden, daß die Macht dieser Tests eher gering ist.

Ein Problem bei dem Engle-Granger-Ansatz besteht darin, daß angenommen wird, es existiere nur eine Kointegrationsbeziehung. Bei drei Variablen, wie in dieser Untersuchung, sind prinzipiell jedoch zwei Kointegrationsbeziehungen möglich. Außerdem sind die Ergebnisse der Engle-Granger-Tests nicht invariant gegenüber der Wahl der Variable auf der linken Seite. Wird im Gegensatz zu Gleichung (3) die kurzfristige Dynamik des Systems einbezogen, kann die Effizienz der geschätzten Kointegrationsparameter erhöht werden. Beim Johansen-Verfahren (vgl. JOHANSEN (1988) und JOHANSEN und JUSELIUS (1990)) wird die Dynamik einbezogen und ein vektorautoregressives Modell geschätzt. In diesem Ansatz ist es möglich, die Anzahl der Kointegrationsvektoren zu bestimmen, die Kointegrationsparameter zu schätzen und ökonomische Restriktionen zu testen. Die Macht dieser multivariaten Kointegrationstests ist nach der Simulationsstudie von GREGORY (1994) bei quadratischen Kontrollproblemen sehr hoch, allerdings wird die Nullhypothese der Nicht-Kointegration zu häufig verworfen, falls sie korrekt ist.

Ausgangspunkt für die durchgeführten Tests bildet das folgende vektorautoregressive Modell der Ordnung $k$ :

$$
Y_{t}=\mu+A_{1} Y_{t-1}+\ldots+A_{k} Y_{t-k}+U_{t} .
$$

Dabei ist $Y_{t}$ ein $n$-dimensionaler Vektor von Variablen, ${ }^{16}$ die integriert von der Ordnung eins sind, $\mu$ ein Vektor von Konstanten, $A_{i}(i=1, \ldots, k)$ eine $(n \times n)$-Koeffizientenmatrix und $U_{t}$ ein multivariater White-Noise-Prozeß. Dieses Modell läßt sich als Vektor-Fehler-Korrektur-Modell darstellen: 


\begin{tabular}{|c|c|c|c|c|c|c|c|c|c|c|c|c|}
\hline & 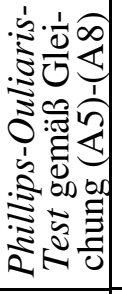 & $\begin{array}{l}N^{2} \\
N^{2}\end{array}$ & 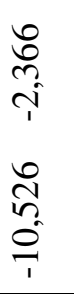 & $\begin{array}{l}\infty \\
\stackrel{\infty}{\infty} \\
\frac{1}{1} \\
\vec{n} \\
\frac{6}{1}\end{array}$ & 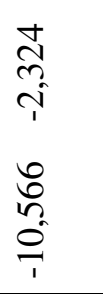 & \begin{tabular}{l}
$\hat{\hat{n}}$ \\
\multirow{1}{1}{} \\
\multirow{2}{0}{} \\
$\infty$ \\
$\dot{+}$
\end{tabular} & 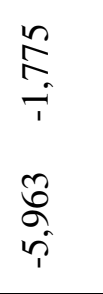 & 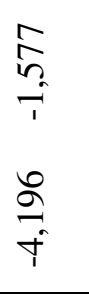 & 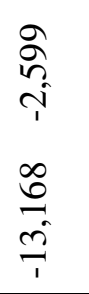 & 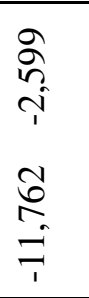 & 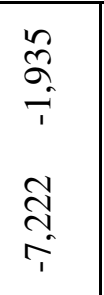 & 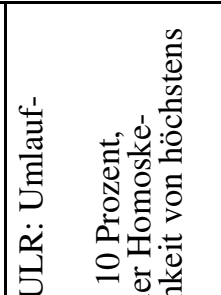 \\
\hline 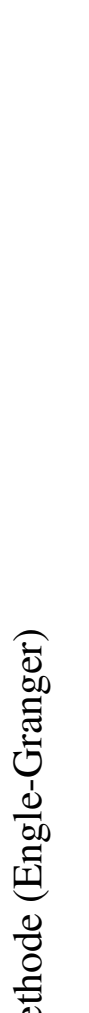 & 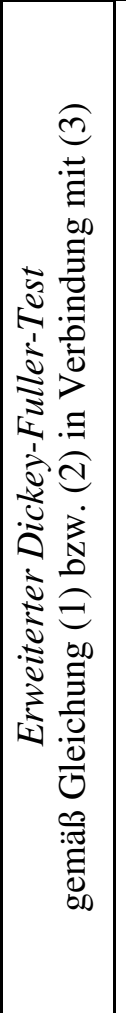 & 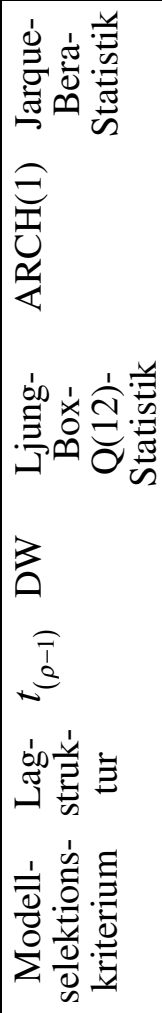 & 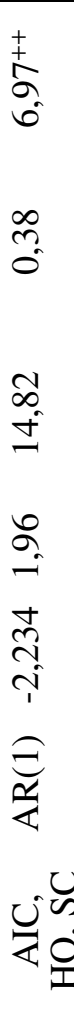 & 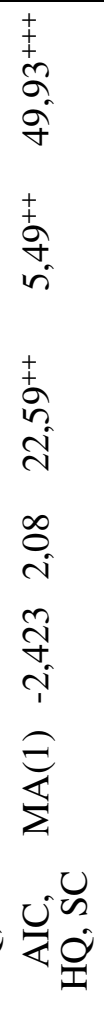 & 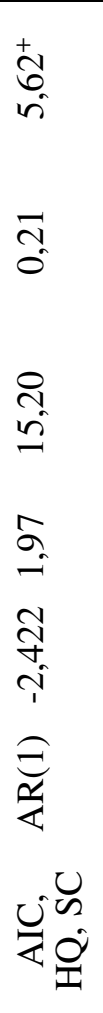 & 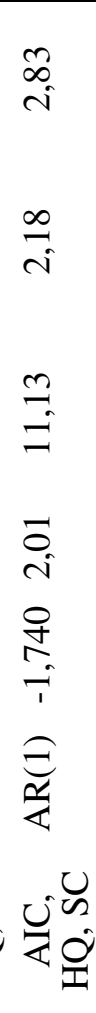 & 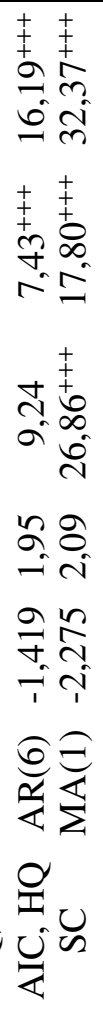 & 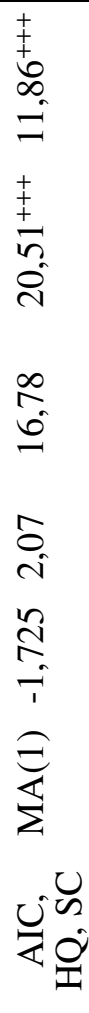 & 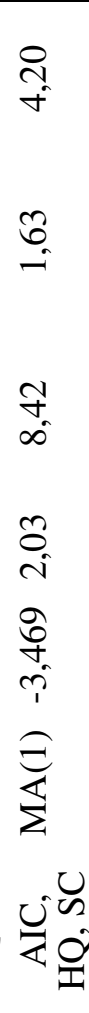 & 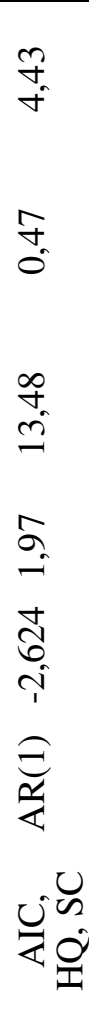 & 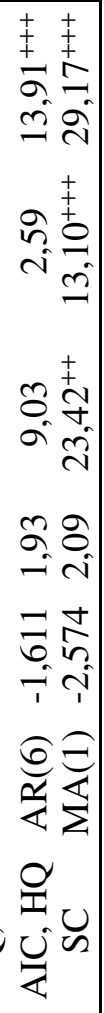 & 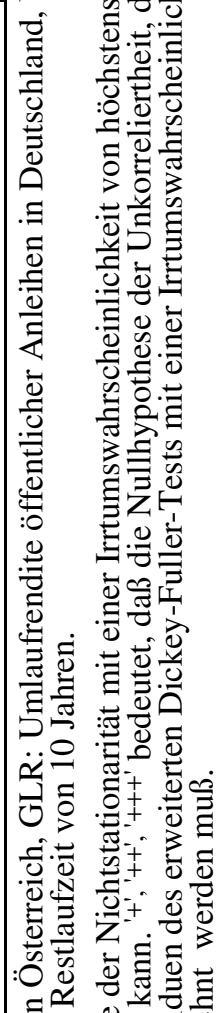 \\
\hline 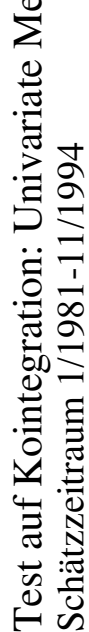 & $\begin{array}{l}\widehat{0} \\
00 \\
\Xi \\
\frac{0}{0} \\
\frac{0}{0}\end{array}$ & 疍 & 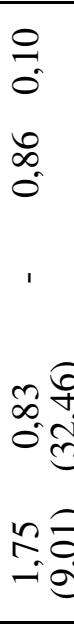 & $\begin{array}{l}n \\
0 \\
0 \\
0 \\
0 \\
0 \\
0 \\
1\end{array}$ & 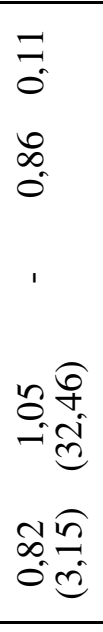 & 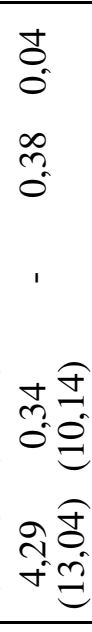 & 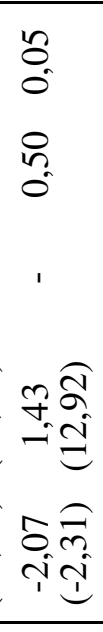 & $\begin{array}{l}\infty \\
2 \\
0 \\
1 \\
=\overparen{\Xi} \\
=0 \\
=0\end{array}$ & 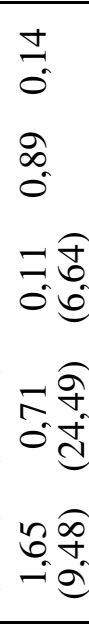 & $\begin{array}{l}m \\
0 \\
0 \\
0 \\
0 \\
0 \\
+\infty \\
0 \\
0 \\
0 \\
1\end{array}$ & $\hat{0}$ & 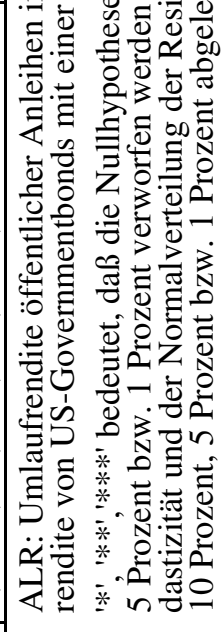 \\
\hline $\begin{array}{l}\ddot{\Xi} \\
\stackrel{0}{0} \\
\stackrel{\Xi}{\Xi} \\
\tilde{\Xi}\end{array}$ & 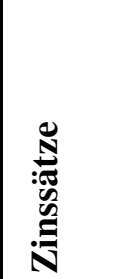 & $\bar{x}$ & $\begin{array}{l}\frac{a}{3} \\
0 \\
\frac{4}{3}\end{array}$ & $\begin{array}{l}\frac{\alpha}{b} \\
\frac{1}{3} \\
\frac{a}{2}\end{array}$ & 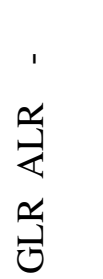 & 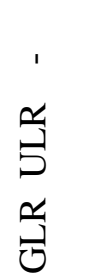 & $\begin{array}{l}\frac{\mathscr{J}}{\mathrm{Z}} \\
\underline{\mathrm{b}} \\
\underline{b}\end{array}$ & 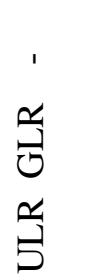 & $\begin{array}{l}\frac{2}{3} \\
5 \\
\frac{1}{3} \\
0 \\
\frac{2}{2} \\
\frac{2}{2}\end{array}$ & 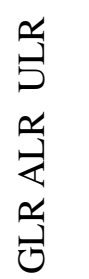 & $\begin{array}{l}\frac{1}{3} \\
0 \\
\frac{1}{3} \\
\frac{1}{2} \\
\frac{1}{3}\end{array}$ & 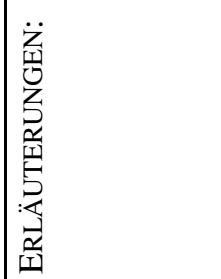 \\
\hline
\end{tabular}




$$
\Delta Y_{t}=\mu+\Gamma_{1} \Delta Y_{t-1}+\ldots+\Gamma_{k-1} \Delta Y_{t-k+1}+\Pi Y_{t-k}+U_{t},
$$

wobei die $\Gamma_{i}=-\left(I-A_{1}-\ldots-A_{i}\right),(i=1, \ldots, k-1)$ die Koeffizentenmatrizen der ersten Differenzen $\Delta Y_{t-i}$ sind, die die kurzfristige Dynamik des Systems beschreiben, und $\Pi=-\left(I-A_{1}-\ldots-A_{k}\right)$ ist die $\left(\begin{array}{lll}n & x & n\end{array}\right)$-Koeffizientenmatrix des verzögerten Niveaus $Y_{t-k}$. Unter der Nullhypothese von $r$ Kointegrationsbeziehungen, $1 \leq r \leq n-1$, kann die Matrix $\Pi$ aufgespalten werden in

$$
\Pi=\alpha \beta^{\prime},
$$

wobei $\alpha$ und $\beta$ (nx r)-Matrizen mit dem Rang $r$ sind, so daß $\beta^{\prime} Y_{t-1}$ stationär ist.

Die $r$ Spalten von $\beta$ sind die linear unabhängigen Kointegrationsvektoren. Die $i$-te Zeile der Gewichtungsmatrix $\alpha$ gibt den Einfluß der $r$ Kointegrationsvektoren auf die Dynamik der $i$-ten Gleichung an. Ist der Rang $r$ der Matrix $\Pi$ gleich null, so gilt $\Pi=0$ und $Y_{t}$ ist ein $n$-dimensionaler Random Walk mit Drift. Ist hingegen der Rang von $\Pi$ gleich $n$, dann ist $\Pi$ regulär und $Y_{t}$ ein stationärer Prozeß.

Um die Zahl der Kointegrationsvektoren zu bestimmen, wird mit Likelihood-RatioTests überprüft, wieviele Eigenwerte $\lambda_{i}$ der Matrix $\Pi$ signifikant von null verschieden sind. Dies ergibt die Zahl der Kointegrationsbeziehungen. Die Nullhypothese, daß höchstens $r$ Kointegrationsvektoren vorliegen, wird mit der von JOHANSEN und JUSELIUS (1990) vorgeschlagenen Trace-Statistik überprüft:

$$
\text { Trace - Statistik }=-T \sum_{i=r+1}^{n} \ln \left(1-\lambda_{i}\right)
$$

wobei $\lambda_{r+1}, \ldots, \lambda_{n}$ die $n-r$ kleinsten quadrierten kanonischen Korrelationskoeffizienten der Residuen der beiden Regressionen $Y_{t-k}$ und $\Delta Y_{t}$ sind, jeweils abhängig von verzögerten Differenzen $\Delta Y_{t-i}(i=1, \ldots, k-1)$ und einem Absolutglied. ${ }^{17}$

Die $\lambda_{\text {max }}$-Statistik

$$
\lambda_{\text {max }}-\text { Statistik }=-T \ln \left(1-\lambda_{r+1}\right)
$$

überprüft die Nullhypothese von $r$ Kointegrationsvektoren gegen die Alternativhypothese von $r+1$ Kointegrationsvektoren. Kritische Werte für beide Teststatistiken finden sich bei JOHANSEN und Juselius (1990, Tabelle A2, S. 208). Beginnend mit $r=0$ kann der Kointegrationsrang sequentiell getestet werden.

Da die Testergebnisse abhängig von der Anzahl der Lags des vektorautoregressiven Modells sein können (vgl. HALL (1991) und CHEUNG und LAI (1993)), wurde dem Vorschlag von LÜTKEPOHL (1991) folgend die Ordnung $k$ in Gleichung (4) mit dem AIC-, HQ- und SC-Modellselektionskriterium festgelegt. ${ }^{18}$ Zusätzlich wurden mit der LjungBox-Q(12)-Statistik, dem ARCH(1)-Test und der Jarque-Bera-Statistik die Residuen der Gleichungen des Vektor-Fehler-Korrektur-Modells untersucht. Falls Autokorrelation in den Residuen nachweisbar war, wurde die Anzahl der Lags zusätzlich erhöht, da bei einer Unterparametrisierung die Nullhypothese von der Trace- und $\lambda_{\max }$-Statistik mit einer deutlich überhöhten Irrtumswahrscheinlichkeit abgelehnt wird. Die Abweichung 
von der Normalverteilung verzerrt dagegen die Testergebnisse nicht sehr stark (vgl. CHEUNG und LAI (1993)).

Die Ergebnisse des multivariaten Kointegrationstests in Tabelle $2 b$ sind sehr eindeutig: Obwohl die Tests eine relativ große Macht haben und dazu neigen, die Nullhypothese zu oft abzulehnen, ${ }^{19}$ konnten zwischen den drei langfristigen Zinssätzen keine Kointegrationsbeziehungen gefunden werden. Dabei wurden sowohl bivariate als auch trivariate Fehler-Korrektur-Modelle benutzt. Die Hypothese der langfristigen deutschen Dominanz, die einen Kointegrationsvektor zwischen dem deutschen und dem österreichischen Zinssatz verlangt, kann somit nicht bestätigt werden.

\subsection{Ergebnisse der Granger-Kausalitätstests}

Die bisherigen Testergebnisse sprechen dafür, daß die drei Zeitreihen integriert von der Ordnung eins, aber nicht kointegriert sind. Die Nullhypothese der Nicht-Kointegration ließ sich jedenfalls mit verschiedenen Verfahren in keinem einzigen Fall verwerfen. Dieses Ergebnis ist bei der Durchführung der Granger-Kausalitätstests zu beachten, denn in einem vektorautoregressiven Modell mit integrierten aber nicht kointegrierten Variablen hat der Wald-Test auf Granger-Kausalität eine Nicht-Standardverteilung gemäß TODA und PHILlIPS (1994, Corollary 2, S. 264). Der sequentiellen Testprozedur von TODA und PHILLIPS (1994) folgend werden daher vektorautoregressive Modelle in ersten Differenzen geschätzt. Die üblichen Hypothesentests sind dann anwendbar. Allerdings können, falls eine Kointegrationsbeziehung vorliegt, aber nicht entdeckt wird, die Kausalitätstests unter gewissen Parameterkonstellationen sehr deutlich in Richtung Ablehnung der korrekten Nullhypothese verzerrt sein, wie TODA und PHILLIPS (1994) in ihrer Simulationsstudie zeigen.

Um die gegenseitige Beeinflussung der drei Zinssätze $\mathrm{zu}$ testen, wird in der vorliegenden Untersuchung das Verfahren von HsiaO $(1979,1981)$ für bi- und trivariate vektorautoregressive Modelle in ersten Differenzen eingesetzt. Dieses Verfahren knüpft direkt an die Definition der Granger-Kausalität an. Im Gegensatz zum philosophischen Kausalitätsbegriff wird bei der Granger-Kausalität (nur) auf die Prognosefähigkeit abgestellt: Eine Variable $X$ wird als granger-kausal zur Variable $Y$ bezeichnet, wenn die Prognose von $Y$ durch die Berücksichtigung der vergangenen Werte von $X$ bei sonst gleicher Informationsmenge verbessert werden kann (vgl. GRANGER (1969) und z. B. KIRCHGÄSSNER (1981)). Diese Definition der Kausalität basiert auf der Grundidee, daß eine Ursache nicht zeitlich nach dem Effekt auftreten kann. ${ }^{20}$ Die Verbesserung der Prognosegüte durch die Berücksichtigung einer zusätzlichen Variable wird beim HsiaoVerfahren anhand von Modellselektionskriterien gemessen. Dabei werden in dieser Untersuchung neben dem Informationskriterium von Akaike, das wie das ursprünglich von HSIAO $(1979,1981)$ benutzte Final-Prediction-Error-Kriterium die wahre Anzahl der Modellparameter überschätzt (vgl. JUDGE u. a. (1985, S. 245)) und damit zu häufig Kausalität anzeigt, mit dem Hannan-Quinn- und Schwarz-Kriterium zwei konsistente Modellselektionskriterien verwendet. 


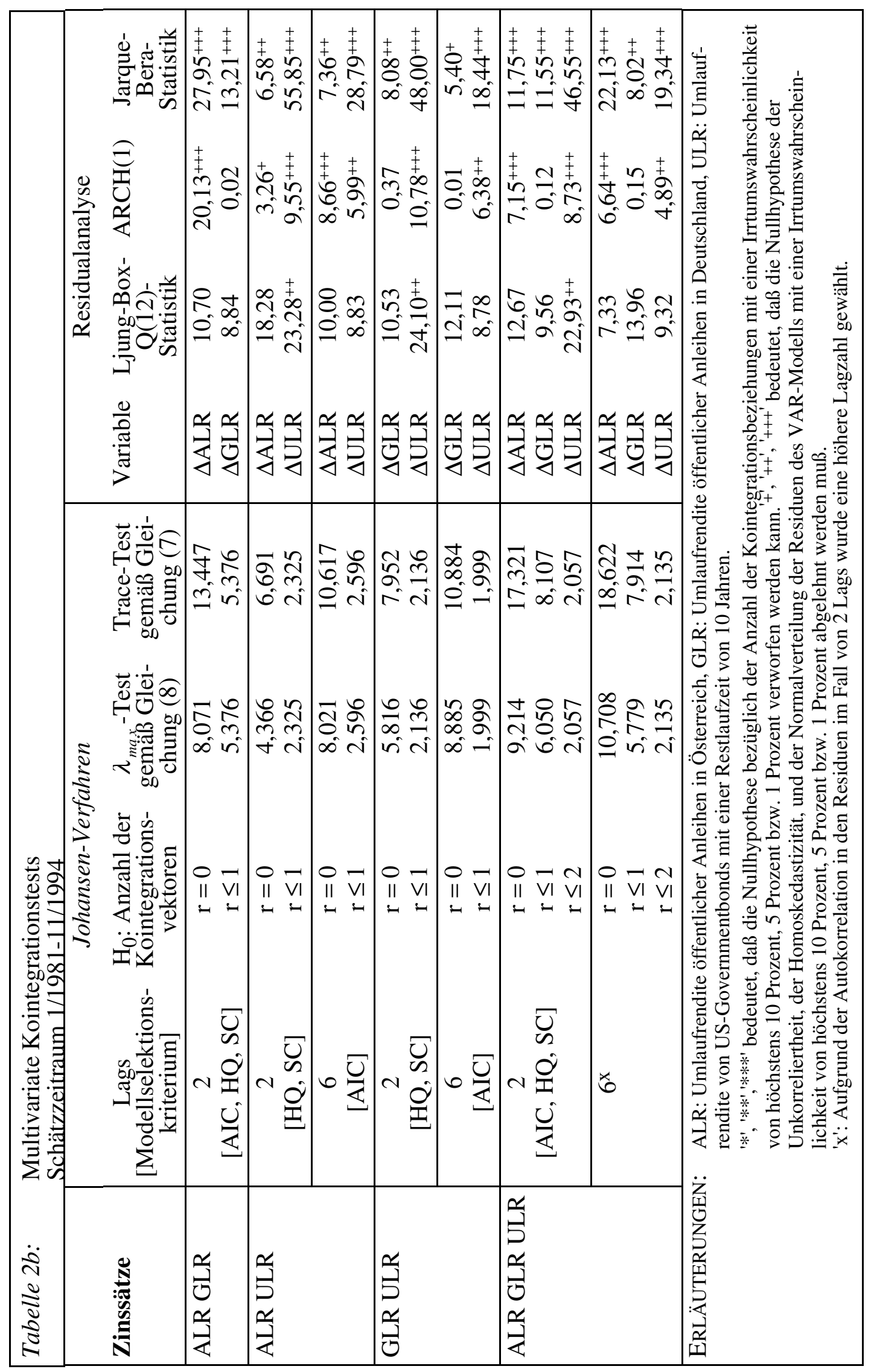


Die von Hsiao vorgeschlagene und hier leicht modifizierte Testprozedur sei für den bivariaten Fall kurz skizziert:

1. Zunächst ist für das vektorautoregressive Modell in ersten Differenzen

$$
\begin{aligned}
& \Delta Y_{t}=\gamma_{1}+\sum_{i=1}^{m_{11}} \gamma_{11, i} \Delta Y_{t-i}+\sum_{i=1}^{m_{12}} \gamma_{12, i} \Delta X_{t-i}+\varepsilon_{1, t} \\
& \Delta X_{t}=\gamma_{2}+\sum_{i=1}^{m_{21}} \gamma_{21, i} \Delta Y_{t-i}+\sum_{i=1}^{m_{22}} \gamma_{22, i} \Delta X_{t-i}+\varepsilon_{2, t}
\end{aligned}
$$

das Minimum des Modellselektionskriteriums und damit die optimale Lagstruktur zu bestimmen. Dabei wird das Modell mit der Methode der kleinsten Quadrate für alle Kombinationen $m_{11}, m_{12}, m_{21}, m_{22} \in[1, \ldots, 6]$ geschätzt und das Minimum des Modellselektionskriteriums bestimmt:

$$
\mathbb{Z}_{1}=\min _{m_{i j}=1, \ldots, 6} \operatorname{KRIT}\left(m_{11}, m_{12}, m_{21}, m_{22}\right) .
$$

2. Daneben wird das vektorautoregressive Modell (9) unter der Restriktion $\gamma_{12, i}=0$ für alle $i$ geschätzt und die optimale Lagstruktur ermittelt. Das Minimum des Modellselektionskriteriums lautet entsprechend:

$$
\mathbb{Z}_{2}=\min _{m_{i j}=1, \ldots, 6} \operatorname{KRIT}\left(m_{11}, 0, m_{21}, m_{22}\right) .
$$

3. Entsprechend wird für das unter der Restriktion $\gamma_{21, i}=0$ für alle $i$ geschätzte Modell das Minimum des Modellselektionskriteriums mit

$$
\mathbb{Z}_{3}=\min _{m_{i j}=1, . ., 6} \operatorname{KRIT}\left(m_{11}, m_{12}, 0, m_{22}\right)
$$

ermittelt.

4. Die in den Schritten 1 bis 3 ermittelte Prognosegüte der Modelle wird verglichen:

- Falls $\mathbb{Z}_{2}>\mathbb{Z}_{1}$, heißt $X$ granger-kausal zu $Y$.

- Falls $\mathbb{Z}_{3}>\mathbb{Z}_{1}$, heißt $Y$ granger-kausal zu $X$.

- Falls $X$ granger-kausal zu $Y$ und ebenfalls $Y$ granger-kausal zu $X$ ist, liegt zwischen $Y$ und $X$ eine Feedbackbeziehung vor.

Die Schritte 1 bis 4 wurden für die drei Modellselektionskriterien $K R I T \in\{\mathrm{AIC}, \mathrm{HQ}, \mathrm{SC}\}$ durchgeführt. Im trivariaten vektorautoregressiven Modell wird die Informationsmenge um die Variable $\Delta Z$ erweitert:

$$
\begin{aligned}
& \Delta Y_{t}=\gamma_{1}+\sum_{i=1}^{m_{11}} \gamma_{11, i} \Delta Y_{t-i}+\sum_{i=1}^{m_{12}} \gamma_{12, i} \Delta X_{t-i}+\sum_{i=1}^{m_{13}} \gamma_{13, i} \Delta Z_{t-i}+\varepsilon_{1, t} \\
& \Delta X_{t}=\gamma_{2}+\sum_{i=1}^{m_{21}} \gamma_{21, i} \Delta Y_{t-i}+\sum_{i=1}^{m_{22}} \gamma_{22, i} \Delta X_{t-i}+\sum_{i=1}^{m_{23}} \gamma_{23, i} \Delta Z_{t-i}+\varepsilon_{2, t} \\
& \Delta Z_{t}=\gamma_{3}+\sum_{i=1}^{m_{31}} \gamma_{31, i} \Delta Y_{t-i}+\sum_{i=1}^{m_{32}} \gamma_{32, i} \Delta X_{t-i}+\sum_{i=1}^{m_{33}} \gamma_{33, i} \Delta Z_{t-i}+\varepsilon_{3, t} .
\end{aligned}
$$


Damit kann untersucht werden, ob Kausalitätsbeziehungen, die im bivariaten Fall festgestellt wurden, möglicherweise auf den Einfluß einer dritten Variable zurückzuführen sind. Aufgrund der Rechnerkapazität wurde die maximal mögliche Anzahl der Lags mit (nur) vier Lags festgelegt und dann das Minimum des Modellselektionskriteriums

$$
\mathbb{Z}_{4}=\min _{m_{i j}=1, \ldots, 4} \operatorname{KRIT}\left(m_{11}, m_{12}, m_{13}, \ldots, m_{33}\right)
$$

bestimmt. Um beispielsweise den granger-kausalen Einfluß von $X$ auf $Y$ zu testen, lautet die Restriktion für Nichtkausalität $\gamma_{12, i}=0$ für alle $i$ und das dazugehörige Minimum des Modellselektionskriteriums

$$
\mathbb{Z}_{5}=\min _{m_{i j}=1, \ldots, 4} \operatorname{KRIT}\left(m_{11}, 0, m_{13}, \ldots, m_{33}\right) .
$$

Falls $\mathbb{Z}_{5}>\mathbb{Z}_{4}$, heißt $X$ granger-kausal zu $Y$.

Entsprechend werden die anderen Kausalitätsbeziehungen getestet. Die Ergebnisse für die bivariaten Modelle sind in Tabelle 3a bis 3c dargestellt, für die trivariaten in Tabelle 4a bis 4c. Sie können wie folgt charakterisiert werden: Unabhängig vom Modell und vom Modellselektionskriterium wird ein granger-kausaler Einfluß der deutschen langfristigen Zinssätze auf die entsprechenden österreichischen Sätze nachgewiesen. Dieses Ergebnis steht im Einklang mit der Teilhypothese der Abhängigkeit der österreichischen von der deutschen Zinsentwicklung. Die Frage der Unabhängigkeit der deutschen von den österreichischen Zinssätzen kann nicht ganz klar beantwortet werden: Die Tests im bivariaten vektorautoregressiven Modell sprechen gegen die Unabhängigkeitsvermutung. Wird hingegen der US-Zins im Informationsset berücksichtigt, dann verschwindet diese Kausalitätsbeziehung. Insofern kann zwischen den deutschen und den österreichischen Finanzmärkten ein asymmetrisches Verhältnis nachgewiesen werden: Der deutsche Zinssatz ist granger-kausal für den österreichischen, aber nicht umgekehrt. Da in allen durchgeführten Untersuchungen die amerikanischen Sätze granger-kausal auf die österreichischen wirken, muß die Teilhypothese von der Isolierung Österreichs vom Rest der Welt klar abgelehnt werden. Darüber hinaus wurden keine eindeutigen Kausalitätsbeziehungen gefunden. ${ }^{21}$ 


\begin{tabular}{|c|c|c|c|c|c|}
\hline \multicolumn{2}{|c|}{ Tabelle 3a: } & \multicolumn{4}{|c|}{$\begin{array}{l}\text { Bivariate VAR-Modelle und Granger-Kausalitätstests } \\
\text { Hsiao-Verfahren mit Akaike's Informationskriterium } \\
\text { gemäß Gleichung (9), Schätzzeitraum 1/1981-11/1994 }\end{array}$} \\
\hline \multirow[t]{2}{*}{ System } & \multicolumn{3}{|c|}{ Lags von } & \multirow[t]{2}{*}{$\mathrm{AIC}_{\mathrm{Min}}$} & \multirow[t]{2}{*}{ Kausalitätsstruktur } \\
\hline & $\triangle \mathrm{ALR}$ & GLR & ULR & & \\
\hline & \multicolumn{5}{|c|}{ VAR-Modell ohne Restriktionen } \\
\hline$\triangle \mathrm{ALR}$ & 1 & 1 & $\circ$ & \multirow{2}{*}{$-7,563$} & \\
\hline$\Delta \mathrm{GLR}$ & 1 & 1 & ○ & & \\
\hline & \multicolumn{5}{|c|}{ VAR-Modelle mit Restriktionen } \\
\hline$\Delta \mathrm{ALR}$ & 6 & 1 & ० & \multirow[t]{2}{*}{$-7,180$} & \multirow[t]{2}{*}{ GLR ist granger-kausal zu ALR } \\
\hline$\Delta \mathrm{GLR}$ & 1 & - & ० & & \\
\hline$\triangle \mathrm{ALR}$ & 1 & 1 & ० & $-7,508$ & ALR ist granger-kausal zu GLR \\
\hline \multirow[t]{2}{*}{$\Delta \mathrm{GLR}$} & - & 1 & ० & & Zwischen GLR und ALR gibt es Feedback \\
\hline & \multicolumn{5}{|c|}{ VAR-Modell ohne Restriktionen } \\
\hline$\Delta \mathrm{ALR}$ & 3 & ० & 2 & \multirow[t]{2}{*}{$-6,061$} & \\
\hline \multirow[t]{2}{*}{$\Delta \mathrm{ULR}$} & 1 & ○ & 6 & & \\
\hline & \multicolumn{5}{|c|}{ VAR-Modelle mit Restriktionen } \\
\hline$\Delta \mathrm{ALR}$ & 3 & ० & 6 & $-5,806$ & ULR ist granger-kausal zu ALR \\
\hline$\Delta \mathrm{ULR}$ & 1 & ○ & - & & \\
\hline$\Delta \mathrm{ALR}$ & 3 & ० & 2 & $-6,027$ & ALR ist granger-kausal zu ULR \\
\hline \multirow[t]{2}{*}{$\Delta \mathrm{ULR}$} & - & o & 2 & & Zwischen ULR und ALR gibt es Feedback \\
\hline & \multicolumn{5}{|c|}{ VAR-Modell ohne Restriktionen } \\
\hline$\Delta \mathrm{GLR}$ & ० & 1 & 5 & \multirow[t]{2}{*}{$-5,267$} & \\
\hline \multirow[t]{2}{*}{$\Delta \mathrm{ULR}$} & ○ & 6 & 2 & & \\
\hline & \multicolumn{5}{|c|}{ VAR-Modelle mit Restriktionen } \\
\hline$\Delta \mathrm{GLR}$ & ○ & 1 & 6 & $-5,193$ & ULR ist granger-kausal zu GLR \\
\hline$\Delta \mathrm{ULR}$ & ○ & 6 & - & & \\
\hline$\Delta \mathrm{GLR}$ & $\circ$ & 3 & 1 & $-5,243$ & GLR ist granger-kausal zu ULR \\
\hline$\Delta \mathrm{ULR}$ & $\circ$ & - & 2 & & Zwischen ULR und GLR gibt es Feedback \\
\hline \multicolumn{6}{|c|}{ ERLÄUTERUNGEN: } \\
\hline \multicolumn{6}{|c|}{$\begin{array}{l}\text { ALR: Umlaufrendite öffentlicher Anleihen in Österreich, GLR: Umlaufrendite öffentlicher Anleih } \\
\text { in Deutschland, ULR: Umlaufrendite von US-Governmentbonds mit einer Restlaufzeit von } \\
10 \text { Jahren. } \\
\text { 'O' bedeutet, daß die entsprechende Variable im System ohne Restriktionen nicht berücksichtigt } \\
\text { wurde. } \\
\text { '-' bedeutet, daß die entsprechenden Koeffizienten auf null restringiert wurden. } \\
\text { AIC }{ }_{\text {Min: Minimum des Informationskriteriums von Akaike. }}\end{array}$} \\
\hline
\end{tabular}




\begin{tabular}{|c|c|c|c|c|c|}
\hline \multicolumn{2}{|c|}{ Tabelle $3 b$ : } & \multicolumn{4}{|c|}{$\begin{array}{l}\text { Bivariate VAR-Modelle und Granger-Kausalitätstests } \\
\text { Hsiao-Verfahren mit dem Hannan-Quinn-Kriterium } \\
\text { gemäß Gleichung (9), Schätzzeitraum 1/1981-11/1994 }\end{array}$} \\
\hline \multirow[t]{2}{*}{ System } & \multicolumn{3}{|c|}{ Lags von } & \multirow{2}{*}{$\mathrm{HQ}_{\mathrm{Min}}$} & \multirow[t]{2}{*}{ Kausalitätsstruktur } \\
\hline & $\Delta \mathrm{ALR}$ & GLR & ULR & & \\
\hline & \multicolumn{5}{|c|}{ VAR-Modell ohne Restriktionen } \\
\hline$\Delta \mathrm{ALR}$ & 1 & 1 & $\circ$ & \multirow[t]{2}{*}{$-7,517$} & \\
\hline \multirow{2}{*}{$\Delta \mathrm{GLR}$} & 1 & 1 & $\circ$ & & \\
\hline & \multicolumn{5}{|c|}{ VAR-Modelle mit Restriktionen } \\
\hline$\Delta \mathrm{ALR}$ & 2 & - & $\circ$ & \multirow[t]{2}{*}{$-7,126$} & \multirow[t]{2}{*}{ GLR ist granger-kausal zu ALR } \\
\hline$\Delta \mathrm{GLR}$ & 1 & 1 & $\circ$ & & \\
\hline$\Delta \mathrm{ALR}$ & 1 & 1 & ० & \multirow[t]{2}{*}{$-7,471$} & ALR ist granger-kausal zu GLR \\
\hline \multirow[t]{2}{*}{$\Delta \mathrm{GLR}$} & - & 1 & ○ & & Zwischen GLR und ALR gibt es Feedback \\
\hline & \multicolumn{5}{|c|}{ VAR-Modell ohne Restriktionen } \\
\hline$\Delta \mathrm{ALR}$ & 3 & $\circ$ & 1 & \multirow[t]{2}{*}{$-5,956$} & \\
\hline \multirow[t]{2}{*}{$\Delta \mathrm{ULR}$} & 1 & ○ & 6 & & \\
\hline & \multicolumn{5}{|c|}{ VAR-Modelle mit Restriktionen } \\
\hline$\Delta \mathrm{ALR}$ & 3 & $\circ$ & - & \multirow[t]{2}{*}{$-5,715$} & \multirow[t]{2}{*}{ ULR ist granger-kausal zu ALR } \\
\hline$\Delta \mathrm{ULR}$ & 1 & ○ & 6 & & \\
\hline$\Delta \mathrm{ALR}$ & 3 & $\circ$ & 1 & \multirow[t]{2}{*}{$-5,959$} & \multirow[t]{2}{*}{ ALR ist nicht granger-kausal zu ULR } \\
\hline \multirow[t]{2}{*}{$\Delta \mathrm{ULR}$} & - & o & 2 & & \\
\hline & \multicolumn{5}{|c|}{ VAR-Modell ohne Restriktionen } \\
\hline$\Delta \mathrm{GLR}$ & $\circ$ & 1 & 1 & \multirow[t]{2}{*}{$-5,176$} & \\
\hline \multirow[t]{2}{*}{$\Delta \mathrm{ULR}$} & $\circ$ & 1 & 2 & & \\
\hline & \multicolumn{5}{|c|}{ VAR-Modelle mit Restriktionen } \\
\hline$\Delta \mathrm{GLR}$ & $\circ$ & 1 & - & $-5,119$ & ULR ist granger-kausal zu GLR \\
\hline$\Delta \mathrm{ULR}$ & $\circ$ & 1 & 2 & & \\
\hline$\Delta \mathrm{GLR}$ & $\circ$ & 1 & 1 & $-5,194$ & GLR ist nicht granger-kausal zu ULR \\
\hline$\Delta \mathrm{ULR}$ & $\circ$ & - & 1 & & \\
\hline \multicolumn{6}{|c|}{ ERLÄUTERUNGEN: } \\
\hline \multicolumn{6}{|c|}{$\begin{array}{l}\text { ALR: Umlaufrendite öffentlicher Anleihen in Österreich, GLR: Umlaufrendite öffentlicher Anleihen } \\
\text { in Deutschland, ULR: Umlaufrendite von US-Governmentbonds mit einer Restlaufzeit von } \\
10 \text { Jahren. } \\
\text { 'O' bedeutet, daß die entsprechende Variable im System ohne Restriktionen nicht berücksichtigt } \\
\text { wurde. } \\
\text { '-' bedeutet, daß die entsprechenden Koeffizienten auf null restringiert wurden. } \\
\text { HQMin: Minimum des Hannan-Quinn-Kriteriums. }\end{array}$} \\
\hline
\end{tabular}




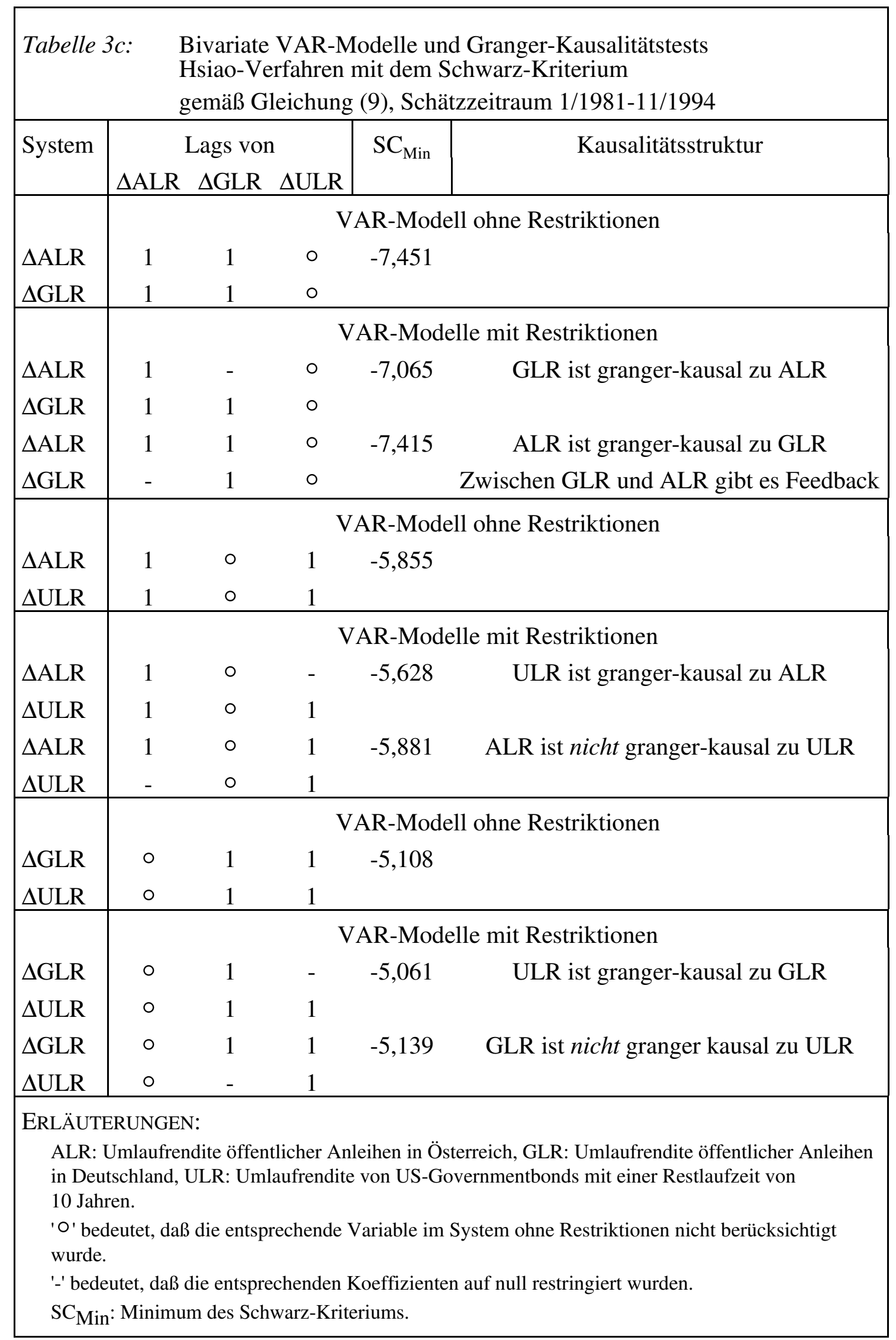




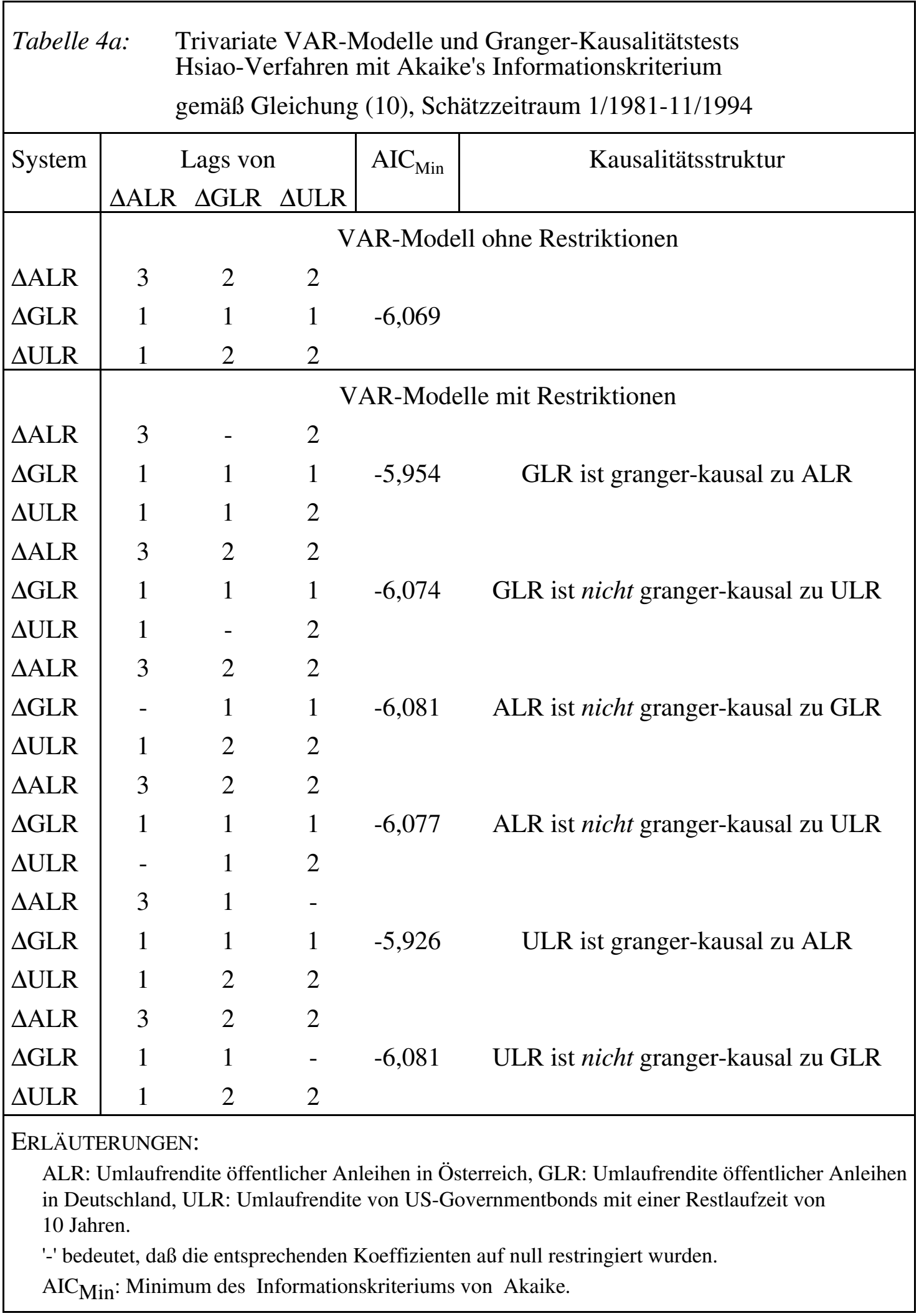




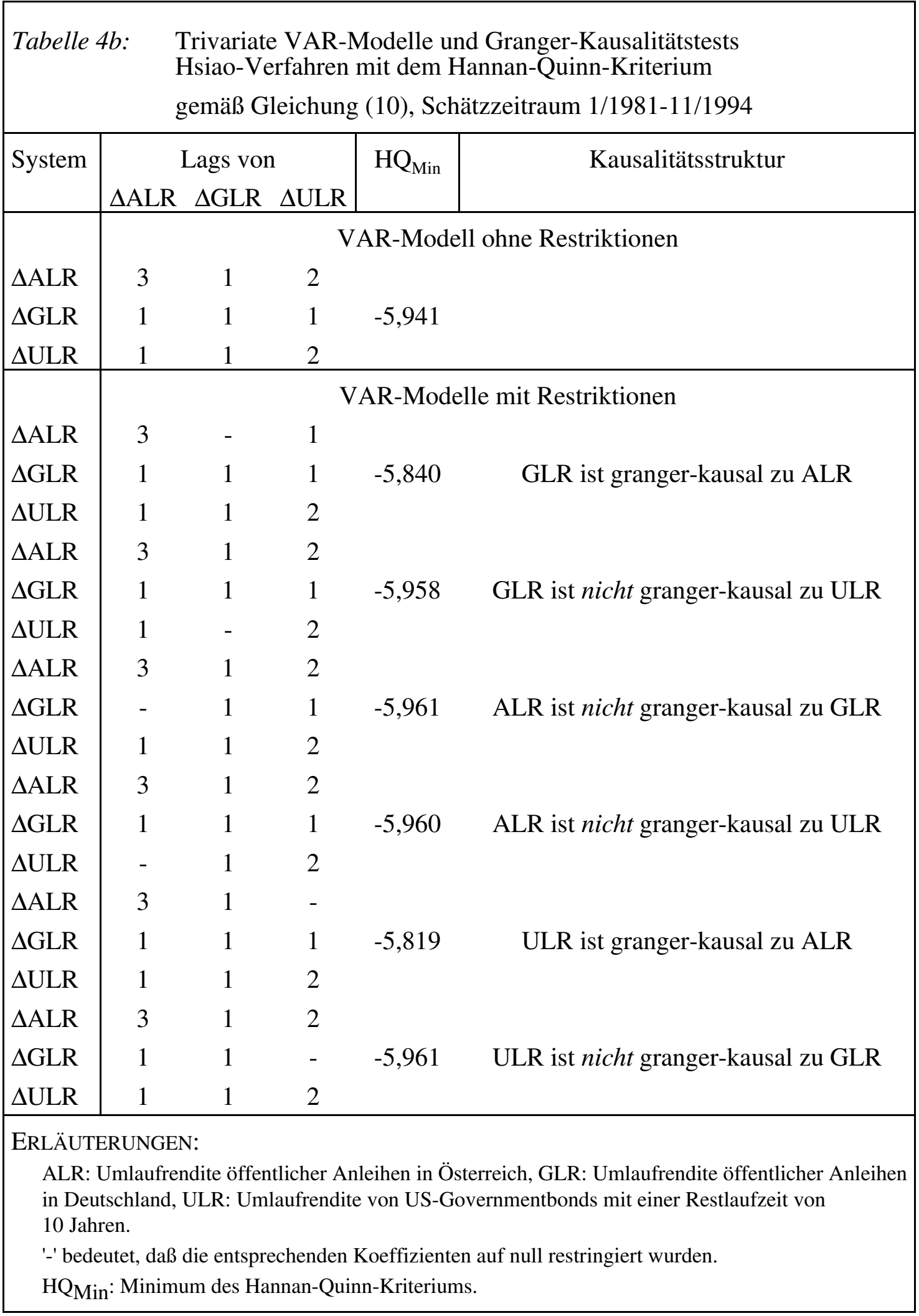




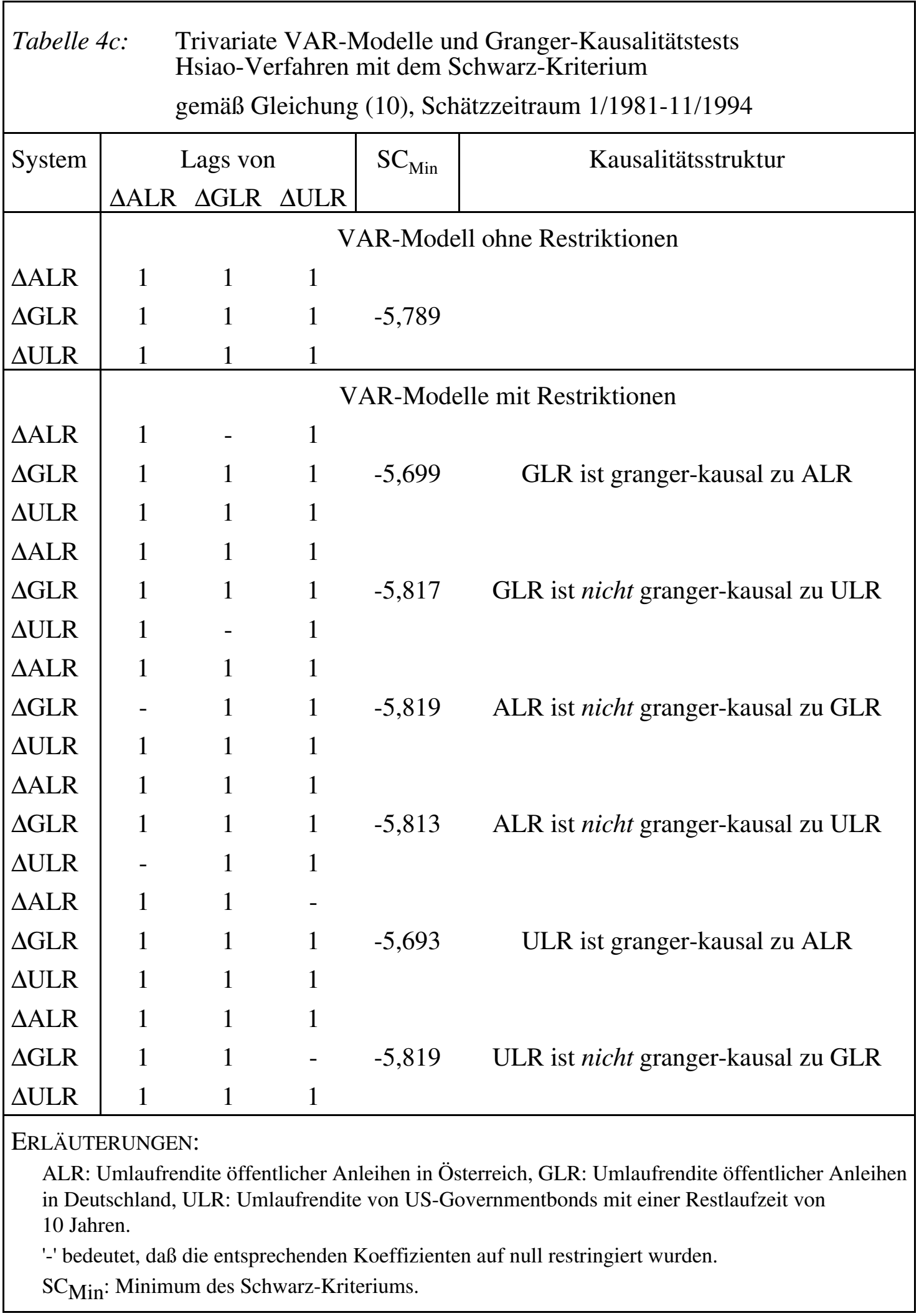


Abbildung 2: Zusammenfassung der Ergebnisse der Granger-Kausalitätstests

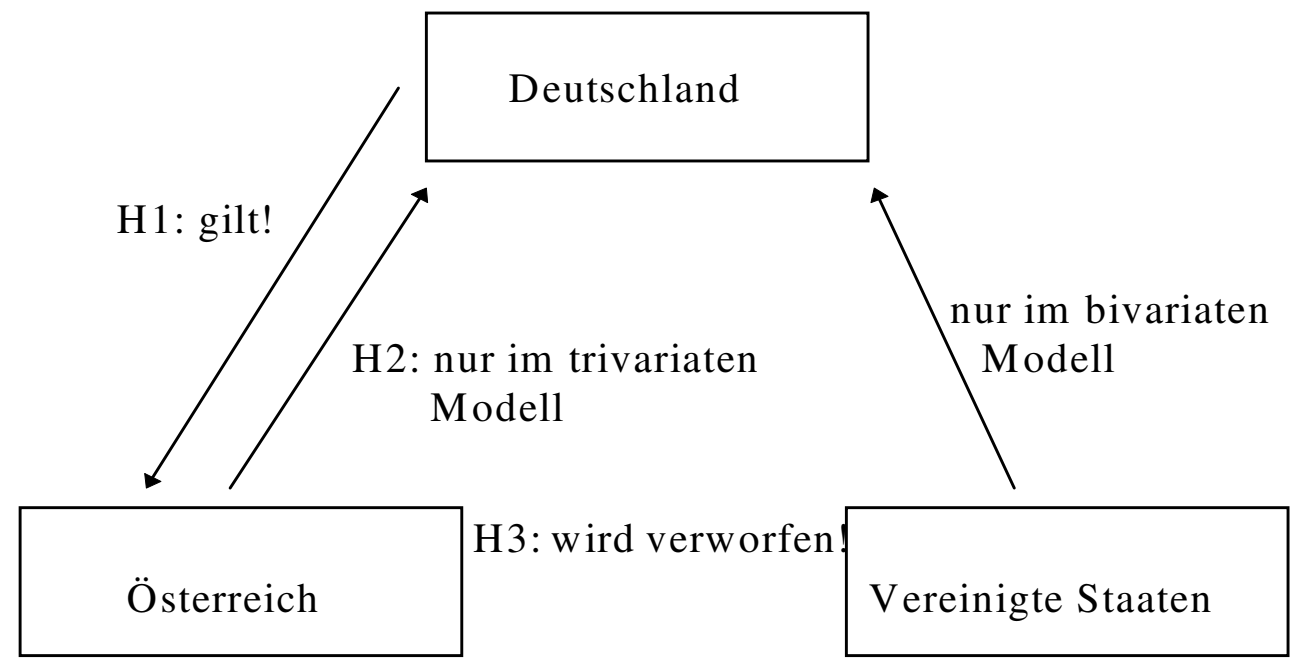

\section{Erläuterungen:}

H1: Abhängigkeit Österreichs von Deutschland

H2: Unabhängigkeit Deutschlands von Österreich

H3: Isolierung Österreichs von den Vereinigten Staaten

Die wesentlichen Ergebnisse des Granger-Kausalitätstests sind in Abbildung 2 zusammengefaßt. Sie zeigen, daß die kurzfristige deutsche Dominanzhypothese, die die gemeinsame Gültigkeit der Hypothesen H1 bis H3 verlangt, verworfen wird. Ergänzende Untersuchungen in bi- und trivariaten Modellen in Niveaus, die bei Vorliegen von Kointegrationsbeziehungen sinnvoll sind, liefern analoge Ergebnisse.

\section{Abschließende Bemerkungen}

In dieser Arbeit wurde die Hypothese der vollen Integration Österreichs in die DMZone, die aufgrund der österreichischen Hartwährungspolitik plausibel erscheint, mit Hilfe der Methodologie der Kointegrations- und Granger-Kausalitätstests für die langfristigen Zinssätze empirisch überprüft. Das Ergebnis ist weitgehend negativ: Langfristige Gleichgewichtsbeziehungen zwischen dem österreichischen und dem deutschen Zinssatz in Form von Kointegration konnten nicht nachgewiesen werden. Zwar ist der deutsche Zinssatz granger-kausal für den österreichischen, doch gilt dies auch für den US-Zinssatz. Das Bestehen unabhängiger Einflüsse der US-amerikanischen Zinssätze (neben den deutschen) auf die österreichischen Zinssätze weist darauf hin, daß die österreichischen Kapitalmärkte nicht von der deutschen Geldpolitik und Zinsentwicklung dominiert werden. Insgesamt muß daher die deutsche Dominanzhypothese für Österreich als falsifiziert betrachtet werden. Österreich ist noch nicht als 
voll in die DM-Zone integriert anzusehen. Untersuchungen mit weiteren ökonomischen Variablen werden zu klären haben, in welchen Bereichen und in welchem Ausmaß die Entwicklung der österreichischen Volkswirtschaft von jener der deutschen divergiert.

Dieses Resultat kann verschiedene Ursachen haben. Beispielsweise kann durch Sondereinflüsse im Untersuchungszeitraum (etwa die deutsche Vereinigung) die langfristige Beziehung zwischen österreichischem und deutschem Zinssatz gestört worden sein. Die Ergebnisse erster Tests auf Strukturbrüche sprechen allerdings eher gegen diese Möglichkeit. Variable Risikoprämien infolge unterschiedlicher Entwicklungen der Inflationserwartungen oder institutionelle Faktoren (etwa die im Untersuchungszeitraum erst allmählich erfolgte Liberalisierung des Kapitalverkehrs) können zu systematischen Verletzungen der Hypothese der ungedeckten Zinsparität führen. Man kann auch argumentieren, daß die Geldpolitik direkt nur die kurzfristigen Zinssätze beeinflussen kann, die langfristigen Zinssätze aber nur indirekt und über den Umweg der Erwartungen der Akteure des privaten Sektors. Da analoge Untersuchungen wie die hier berichteten mit Drei-Monats-Geldmarktsätzen Kointegration zwischen Österreich und Deutschland anzeigen, kann vermutet werden, daß im Untersuchungszeitraum die Dominanz der deutschen Geldpolitik bereits in den kurzfristigen, (noch) nicht aber in den langfristigen österreichischen Zinssätzen wirksam wird. Die Konvergenz der langfristigen Zinssätze ist aber der eigentliche „Lackmustest" für die Glaubwürdigkeit einer Wechselkursbindung auf den Finanzmärkten. Daher können die Ergebnisse unserer Untersuchung dahingehend interpretiert werden, daß der durch die Hartwährungspolitik angestrebte Reputationsaufbau im Fall Österreichs mindestens zehn Jahre gedauert hat. Wirtschaftspolitisch stellt sich dann die Frage, ob nicht eine ähnliche (oder längere) Zeitperiode erforderlich sein wird, um die Glaubwürdigkeit einer künftigen europäischen Gemeinschaftswährung zu etablieren. Eine Antwort darauf liegt jedoch außerhalb der Möglichkeiten des vorliegenden Beitrags.

\section{Anhang: Phillips-Perron- und Phillips-Ouliaris-Test}

Um den von PHILLIPS (1987) und PHILLIPS und PERRON (1988) vorgeschlagenen $Z_{\rho}$ - und $Z_{t}$-Test durchzuführen, wird mit der Methode der kleinsten Quadrate (OLS) die Gleichung

$$
Y_{t}=\alpha+\rho Y_{t-1}+U_{t}
$$

geschätzt. Die Phillips-Perron- $Z_{\rho}$-Statistik ist dann wie folgt definiert:

$$
Z_{\rho}=T(\hat{\rho}-1)-\frac{1}{2}\left(S_{T l}^{2}-S_{U}^{2}\right)\left\{T^{-2} \sum_{t=2}^{T}\left(Y_{t-1}-\bar{Y}_{-1}\right)^{2}\right\}^{-1}
$$

wobei $S_{U}^{2}$ die Varianz der Residuen $\hat{U}_{t}$ ist und

$$
S_{T l}^{2}=\frac{1}{T} \sum_{t=1}^{T} \hat{U}_{t}^{2}+\frac{2}{T} \sum_{j=1}^{l} \omega_{j l} \sum_{t=j+1}^{T} \hat{U}_{t} \hat{U}_{t-j}
$$


mit den Gewichten $\omega_{j l}=\{1-j(l+1)\}$, die bewirken, daß $S_{T l}^{2}$ nicht negativ werden kann.

Unter der Nullhypothese $H_{0}:|\rho|=1$ hat die $Z_{\rho}$-Statistik asymptotisch die gleiche Verteilung wie die Teststatistik $I\left(\rho_{\mu}-1\right)$ von Dickey und Fuller bei identisch normalverteilten Residuen. Damit können die kritischen Werte von FULLER (1976, Tabelle 8.5.1, S. 371) benutzt werden.

Der von Phillips und Perron modifizierte Dickey-Fuller-'t-Test' lautet:

$$
Z_{t}=(\hat{\rho}-1)\left(S_{U} / S_{T l}\right)-\frac{1}{2}\left(S_{T l}^{2}-S_{U}^{2}\right)\left\{S_{T l}^{2} T^{-2} \sum_{t=2}^{T}\left(Y_{t-1}-\bar{Y}_{-1}\right)^{2}\right\}^{-1 / 2},
$$

wobei $S_{T l}^{2}$ gemäß Gleichung (A3) definiert ist. Dabei gelten die kritischen Werte von $\hat{\tau}_{\mu}$ (vgl. z. B. FuLLER (1976, Tabelle 8.5.2, S. 373)).

Der von PHILliPS und OULIARIS (1990) vorgeschlagene $Z_{\rho}$-Test wird durchgeführt, indem für die Residuen $U_{t}$ der einfachen kointegrierenden Gleichung (3) entsprechend dem Phillips-Perron-Einheitswurzeltest die Gleichung

$$
\hat{U}_{t}=\rho \hat{U}_{t-1}+\varepsilon_{t}
$$

geschätzt und die $Z_{\rho}$-Statistik

$$
Z_{\rho}=T(\hat{\rho}-1)-\frac{1}{2}\left(S_{T l}^{2}-S_{\varepsilon}^{2}\right)\left\{T^{-2} \sum_{t=2}^{T} \hat{U}_{t-1}^{2}\right\}^{-1}
$$

mit der Varianz der Residuen $S_{\varepsilon}^{2}$ und

$$
S_{T l}^{2}=\frac{1}{T} \sum_{t=1}^{T} \hat{\varepsilon}_{t}^{2}+\frac{2}{T} \sum_{j=1}^{l} \omega_{j l} \sum_{t=j+1}^{T} \hat{\varepsilon}_{t} \hat{\varepsilon}_{t-j}
$$

berechnet wird. Die Gewichte wurden wieder mit $\omega_{j l}=\{1-j(l+1)\}$ festgelegt und $l$ entsprechend mit $l=\operatorname{Int}\left\{4(T / 100)^{1 / 4}\right\}$. Kritische Werte für einen Beobachtungsumfang von 50 bis 250 finden sich bei HaUG (1992, Tabelle 2, S. 477) und für einen von 500 bei PHILLIPS und OULIARIS (1990, Tabelle Ia, S.189).

Die $Z_{t}$-Phillips-Ouliaris-Teststatistik lautet

$$
Z_{t}=\left\{\sum_{t=2}^{T} \hat{U}_{t-1}^{2}\right\}^{1 / 2} \frac{(\hat{\rho}-1)}{S_{T l}}-\frac{1}{2}\left(S_{T l}^{2}-S_{\varepsilon}^{2}\right)\left\{S_{T l}^{2} T^{-2} \sum_{t=2}^{T} \hat{U}_{t-1}^{2}\right\}^{-1 / 2},
$$

wobei $\hat{\rho}$ gemäß Gleichung (A5) und $S_{T l}^{2}$ gemäß Gleichung (A7) definiert ist und $S_{\varepsilon}^{2}$ die Varianz der Residuen $\hat{\varepsilon}_{t}$ aus Gleichung (A5) darstellt. $Z_{t}$ hat asymptotisch die gleiche Verteilung wie der von ENGLE und GRANGER (1987) vorgeschlagene erweiterte Dickey- 
Fuller-Test auf Kointegration. Entsprechend können die kritischen Werte von MACKINNON (1991) benutzt werden.

\section{Anmerkungen}

* Frühere Versionen dieser Arbeit wurden auf den Österreichischen Statistik-Tagen 1996 in Wien, 10. - 12. April 1996, im Diplomandenseminar zur Statistik und Ökonometrie des Fachbereichs Wirtschaftswissenschaften der Universität Osnabrück am 21. Mai 1996 sowie auf der Jahrestagung 1996 des Vereins für Socialpolitik in Kassel, 25. - 27. September 1996, vorgetragen. Wir danken den Diskussionsteilnehmern dieser Präsentationen, Gebhard Kirchgässner (Universität St. Gallen), Dieter Nautz (FU Berlin), Erich W. Streißler (Universität Wien), Peter Winker (Universität Konstanz), Jochen Jungeilges, Lothar Knüppel, Gerald Musiol, Ralf Pauly, Jörg Schimmelpfennig, Axel Sieker und Mareike Vollbrecht (jeweils Universität Osnabrück) sowie zwei anonymen Gutachtern dieser Zeitschrift für wertvolle Anregungen. Die Verantwortung für verbliebene Unzulänglichkeiten tragen wir.

1 Vgl. dazu unter anderem HANDLER (1989), NECK (1993).

2 Vgl. dazu HolzMANN und NECK (1993).

3 Vor allem WinCKLER (1986); vgl. auch WinCKLER und AMANN (1986), WiNCKLER (1991).

4 Vgl. dazu für die Schweiz KIRCHGÄSSNER und WOLTERS (1991).

5 Die Restlaufzeit der einbezogenen österreichischen Anleihen beträgt mindestens ein Jahr und die der deutschen mindestens drei Jahre. Langfristige Zinssätze mit genau gleicher Restlaufzeit für die drei Währungen stehen nur für einen sehr kurzen Zeitraum zur Verfügung.

6 Quelle: OECD Statistics, Interest Rates on International and Domestic Markets, Monthly Update, December 1994.

7 Es wurden hier die kritischen Werte von MACKINNON (1991) benutzt.

8 Falls der MA(1)-Koeffizient $\alpha$ sehr groß oder relativ klein ist, führt nach Agiakloglou und Newbold (1992, S. 479) der Einsatz von Modellselektionskriterien zu unbefriedigenden Ergebnissen. Bei den durchgeführten Tests tritt dieses Problem allerdings nicht auf. Die geschätzten MA(1)-Koeffizienten liegen alle im Bereich zwischen 0,29 und 0,69.

9 Die Durbin-Watson-Statistik ist jedoch bei verzögerten endogenen Variablen verzerrt und hat eine geringe Macht.

10 Allerdings zeigt die Ljung-Box-Q-Statistik im Fall der langfristigen US-Zinssätze eine signifikante Autokorrelation in den Residuen an. Wird in Gleichung (3) ein höherer AR-Prozeß gewählt, z. B. mit $k=3$, dann ist die Autokorrelation nicht mehr signifikant. Die Aussage des Dickey-Fuller-Tests ändert sich nicht.

11 Die beiden Teststatistiken sind im Anhang dargestellt. 
12 Im Fall mit nur zwei Variablen wird $\beta_{2}$ gleich null gesetzt.

13 Die kritischen Werte sind tabelliert bei ENGLE und GRANGER (1987, Tabelle II, S. 269).

14 Kritische Werte finden sich bei MACKINNON (1991).

15 Die Berechnung der Teststatistiken befinden sich im Anhang.

16 Zum Beispiel ist beim dreidimensionalen Vektorautoregressionsmodell $Y=($ ALR, GLR, ULR)'.

17 Zur Berechnung der $\lambda_{i}$ vgl. JoHANSEN und Juselius (1990, S. 177, Fußnote 3).

18 Dabei wird nach PAULSEN (1984) die Anzahl der Lags auch bei einem VAR-Modell mit Einheitswurzeln von dem HQ- und SC-Kriterium konsistent geschätzt, während das AIC-Kriterium die Ordnung überschätzt.

19 Nach der Response-Surface-Analyse von CHEUNG und LAI (1993, S. 316-319) steigt die Verzerrung des Johansen-Trace- und $\lambda_{\max }$-Tests bei gegebenem Stichprobenumfang mit der Anzahl der Lags $k$ und der Dimension $n$ des vektorautoregressiven Prozesses.

20 Wird hingegen die Ursache aufgrund von Erwartungen antizipiert, dann können Effekte bereits zeitlich vor der eigentlichen Ursache auftreten. Das Konzept der Granger-Kausalität ist in diesem Fall nicht anwendbar. Vgl. hierzu GERHARDS (1994, S. 99 f.).

21 Bei der (nur) mit dem AIC-Kriterium im bivariaten Modell gefundenen Kausalität von den deutschen und österreichischen Sätzen zu den US-Zinssätzen (vgl. Tabelle 3a) sollte beachtet werden, daß das AIC-Kriterium im Erwartungswert die Modellparameter überschätzt und damit zu häufig Kausalität anzeigt. Mit den beiden anderen konsistenten Modellselektionskriterien ließen sich diese Kausalitätsaussagen nicht bestätigen.

\section{Literatur}

Agiakloglou, C., Newbold, P. (1992). Empirical Evidence on Dickey-Fuller-Type Tests. Journal of Time Series Analysis 13, 471-483.

Cheung, Y.-W., LaI, K. S. (1993). Finite-Sample Sizes of Johansen's Likelihood Ratio Tests for Cointegration. Oxford Bulletin of Economics and Statistics 55, 313-328.

Engle, R. F., Granger, C. W. J. (1987). Co-Integration and Error Correction: Representation, Estimation, and Testing. Econometrica 55, 251-267.

Fuller, W. A. (1976). Introduction to Statistical Time Series. New York: John Wiley and Sons. 
GERHARDS, T. (1994). Theorie und Empirie flexibler Wechselkurse. Eine ökonometrische Untersuchung mit Methoden der Kointegration und der multivariaten Zeitreihenanalyse. Heidelberg: Physica-Verlag.

Giavazzi, F., Giovannini, A. (1989). Limiting Exchange Rate Flexibility. The European Monetary System. Cambridge, MA: MIT Press.

GRANGER, C. W. J. (1969). Investigating Causal Relations by Econometric Models and Cross-spectral Methods. Econometrica 37, 424-438.

GrANGER, C. W. J. (1986). Developments in the Study of Cointegrated Economic Variables. Oxford Bulletin of Economics and Statistics 48, 213-228.

Gregory, A. W. (1994). Testing for Cointegration in Linear Quadratic Models. Journal of Business \& Economic Statistics 12, 347-360.

Hagen, J. v./Fratianni, M. (1990). German Dominance in the EMS: Evidence from Interest Rates. Journal of International Money and Finance 9, 358-375.

Hall, A. (1994). Testing for a Unit Root in Time Series with Pretest Data-Based Model Selection. Journal of Business \& Economic Statistics 12, 461-470.

HaLl, S. G. (1991). The Effect of Varying Length VAR Models on the Maximum Likelihood Estimates of Cointegrating Vectors. Scottish Journal of Political Economy 38, 317-323.

HANDLER, H. (1989). Grundlagen der österreichischen Hartwährungspolitik. Wien: Manz.

Hassler, U. (1993). Unit Root Tests: The Autoregressive Approach in Comparison with the Periodogram Regression. Statistical Papers 34, 67-82.

Haug, A. A. (1992). Critical Values for the $Z_{\alpha}$-Phillips-Ouliaris Test for Cointegration. Oxford Bulletin of Economics and Statistics 54, 473-480.

Hochreiter, E., WincKler, G. (1995). The Advantages of Tying Austria's Hands: The Success of the Hard Currency Strategy. European Journal of Political Economy 11, 83-111.

HolzMAnN, R., NECK R. (Hrsg.) (1993). Konjunktureffekte der österreichischen Budgetpolitik. Wien: Manz.

HsiaO, C. (1979). Autoregressive Modelling of Canadian Money and Income Data. Journal of the American Statistical Association 74, 553-560.

HsiaO, C. (1981). Autoregressive Modelling and Money-Income Causality Detection, Journal of Monetary Economics 7, 85-106.

JohAnsen, S. (1988). Statistical Analysis of Cointegration Vectors. Journal of Economic Dynamics and Control 12, 231-254. 
JoHANSEN, S., JusElius, K. (1990). Maximum Likelihood Estimation and Inference on Cointegration - With Applications to the Demand for Money. Oxford Bulletin of Economics and Statistics 52, 169-210.

Judge, G. G., Griffiths, W. E., HiLl, R. C., LÜTKePOHL, H., LeE, T.-C. (1985). The Theory and Practice of Econometrics. Second Edition. New York: John Wiley and Sons.

KIRCHGÄSSNER, G. (1981). Einige neuere statistische Verfahren zur Erfassung kausaler Beziehungen zwischen Zeitreihen. Göttingen: Vandenhoeck und Ruprecht.

KIRCHGÄSSNER, G., WOLTERS, J. (1991). Die Abhängigkeit der schweizerischen von der europäischen und amerikanischen Zinsentwicklung. Empirische Ergebnisse für die achtziger Jahre. Schweizerische Zeitschrift für Volkswirtschaft und Statistik 127, 631-646.

KIRCHGÄSSNER, G., Wolters, J. (1993). Does the DM Dominate the Euro Market? An Empirical Investigation. Review of Economics and Statistics 75, 773-778.

KIRCHGÄSSNER, G., WOLTERS, J. (1995). Interest Rate Linkages in Europe Before and After the Introduction of the Monetary System. Some Empirical Results. Empirical Economics 20, 435-454.

LÜTKEPOHL, H. (1991). Analyse trendbehafteter multipler Zeitreihen. Allgemeines Statistisches Archiv 75, 103-123.

MacKinnon, J. G. (1991). Critical Values for Co-integration Tests. In: Long-run Economic Relationships (R. F. Engle und C. W. J. Granger, Hrsg.), 267-276. Oxford: Oxford University Press.

MacKinnon, J. G. (1994). Approximate Asymptotic Distribution Functions for UnitRoot and Cointegration Tests. Journal of Business \& Economic Statistics 12, 167176.

NECK, R. (1993). Österreich und das Europäische Währungssystem. In: Österreich und Europa 1993. Herausforderungen der europäischen Integration für eine kleine offene Volkswirtschaft (R. Neck, F. Schneider, Hrsg.), 183-207. Wien: Manz.

PAulsen, J. (1984). Order Determination of Multivariate Autoregressive Time Series with Unit Roots. Journal of Time Series Analysis 5, 115-127.

PhILliPS, P. C. B. (1986). Understanding Spurious Regressions in Econometrics. Journal of Econometrics 33, 311-340.

PhILLIPS, P. C. B. (1987). Time Series Regressions with a Unit Root. Econometrica 55, 277-301.

PhILliPs, P. C. B., Ouliaris, S. (1990). Asymptotic Properties of Residual Based Tests for Cointegration. Econometrica 58, 165-193. 
Phillips, P. C. B., Perron, P. (1988). Testing for a Unit Root in Time Series Regression, Biometrika 75, 335-346.

SAID, S. E., DickeY, D. A. (1985). Hypothesis Testing in ARIMA(p,1,q) Models. Journal of the American Statistical Association 80, 369-374.

SCHWERT, G. W. (1987). Effects of Model Specification on Tests for Unit Roots in Macroeconomic Data. Journal of Monetary Economics 20, 73-103.

Schwert, G. W. (1989). Tests for Unit Roots: A Monte Carlo Investigation. Journal of Business \& Economic Statistics 7, 147-159.

Sims, C. A., Stock, J. H., Watson, M. W. (1990). Inference in Linear Time Series Models with Some Unit Roots. Econometrica 58, 113-144.

TIEDE, M. (1991). Unsichere statistische Befunde von Granger-Kausalitätsbeziehungen. Jahrbücher für Nationalökonomie und Statistik 208, 98-102.

ToDA, H. Y., Phillips, P. C. B. (1994). Vector Autoregression and Causality: A Theoretical Overview and Simulation Study. Econometric Reviews 13, 259-285.

WiNCKLER, G. (1986). Strategische Probleme der österreichischen Hartwährungspolitik. Quartalshefte der Girozentrale 21 (3), 55-69.

WincKLER, G. (1991). Exchange Rate Appreciation as a Signal of a New Policy Stance, IMF Working Paper, Washington, D.C.

Winckler, G., Amann, E. (1986). Exchange Rate Policy in the Presence of a Strong Trade Union. Journal of Economics, Supplement 5, 259-280.

Adressen der Autoren:

Dipl.-Kfm. Johannes Jaenicke

Fachbereich Wirtschaftswissenschaften

Universität Osnabrück

D-49069 Osnabrück

E-Mail: jaenicke@oec.uni-osnabrueck.de

o. Univ. Prof. Dr. Reinhard Neck

Institut für Wirtschaftswissenschaften

Universität Klagenfurt

Universitätsstraße 65-67

A-9020 Klagenfurt

E-Mail: reinhard.neck@uni-klu.ac.at 